\title{
Caristi Type Coincidence Point Theorem in Topological Spaces
}

\author{
Jiang Zhu, ${ }^{1}$ Lei Wei, ${ }^{1}$ and Cheng-Cheng $\mathrm{Zhu}^{2}$ \\ ${ }^{1}$ School of Mathematics and Statistics, Jiangsu Normal University, Xuzhou 221116, China \\ ${ }^{2}$ School of Mathematics and Statistics, Lanzhou University, Lanzhou, Gansu 730000, China \\ Correspondence should be addressed to Jiang Zhu; jzhuccy@163.com
}

Received 6 June 2013; Revised 7 August 2013; Accepted 13 August 2013

Academic Editor: Wei-Shih Du

Copyright (C) 2013 Jiang Zhu et al. This is an open access article distributed under the Creative Commons Attribution License, which permits unrestricted use, distribution, and reproduction in any medium, provided the original work is properly cited.

\begin{abstract}
A generalized Caristi type coincidence point theorem and its equivalences in the setting of topological spaces by using a kind of nonmetric type function are obtained. These results are used to establish variational principle and its equivalences in $d$-complete spaces, bornological vector space, seven kinds of completed quasi-semimetric spaces equipped with $Q$-functions, uniform spaces with $q$-distance, generating spaces of quasimetric family, and fuzzy metric spaces.
\end{abstract}

\section{Introduction}

Caristi's fixed-point theorem $[1,2]$ and its equivalences, Ekeland variational principle $[3,4]$, and Takahashi minimization theorem are forceful tools in nonlinear analysis, control theory, and global analysis; see, for example, [3-5]. In the last two decades, Caristi's fixed-point theorem and Ekeland variational principle have been generalized and extended in several directions. About these, one can refer to, for example, [1-32] and the references therein. In particular, in [25], a very general Ekeland variational principle and Caristi's fixed-point theorem are presented, which give a unified approach to three classes of Ekeland type variational principle: in the first class, the underlying space is a sequentially complete uniform space (or equivalently, a sequentially complete F-type topological space), and the perturbation involves a family of topology generating pseudometrics (or quasimetrics); in the second class, the underlying space is a locally complete locally convex space (resp., a locally complete locally $p$-convex space), and the perturbation involves a family of topology generating seminorms (resp., topology generating $p$-homogeneous $F$ pseudonorms) or involves a single Minkowski functional; in the third class, the underlying space is a complete metric space, and the perturbation involves a $w$-distance or a $\tau$ function. On the other hand, Banach fixed-point theorem has been extended to large class of nonmetric spaces which included $d$-complete topological spaces, symmetric spaces, and quasimetric spaces (see, e.g., [33-35]). But to our knowledge, neither Ekeland's variational principle nor any of its equivalents have been established in such $d$-complete topological spaces.

Motivated by the aforementioned works, we attempt to give a unified approach to the previous works. A generalized Caristi type coincidence point theorem in the setting of topological spaces by using a kind of nonmetric type function is proved. As an application of this Caristi's coincidence point theorem, an Ekeland type variational principle and its equivalences in the setting of topological spaces are obtained. Also, these results present Caristi type coincidence point theorem, variational principle, and its equivalences in $d$-complete topological spaces. Moreover, these results are used to establish variational principle and its equivalences in bornological vector space, seven kinds of completed quasi-semimetric spaces equipped with $Q$-functions, uniform spaces with $q$-distance, generating spaces of quasimetric family, and fuzzy metric spaces. The results of this paper uniformly extend and generalize the corresponding results appeared in the literature $[1-4,6-13,15,25,26,28,30,32]$.

\section{Caristi Type Coincidence Point Theorem}

The primary goal of this section is to establish two equivalent generalized Caristi type coincidence point theorems in the setting of topological spaces by using a kind of nonmetric type function. As an application of these Caristi's coincidence 
point theorems, equivalent generalized Caristi type common fixed point theorem, Caristi type fixed point theorem for set valued, Caristi type fixed point theorem for single-valued map, Ekeland type variational principles and its equivalences in the setting of topological spaces are obtained. To establish our main results, we need the following definitions.

Definition 1 (see [15]). Let $(X, \tau)$ be a topological space. An extended real-valued function $f: X \rightarrow(-\infty,+\infty]$ is said to be sequentially lower monotone if for every sequence $\left\{x_{n}\right\}$ converging to $x$ and satisfying

$$
f\left(x_{1}\right) \geq f\left(x_{2}\right) \geq \cdots \geq f\left(x_{n}\right) \geq \cdots
$$

we have $f(x) \leq f\left(x_{n}\right)$, for each $n$.

Definition 2. Let $(X, \tau)$ be a topological space and $p$ : $X \times X \rightarrow[0,+\infty]$ a function. A proper function $f:$ $X \rightarrow(-\infty,+\infty]$ (i.e., $f(x)$ is not identically to $+\infty)$ is said to be sequentially lower monotone with respect to $p$ (in short, sequentially lower monotone with respect to $p$ ) if for any sequence $\left\{x_{n}\right\}$ in $X$ satisfying $\sum_{n=1}^{\infty} p\left(x_{n}, x_{n+1}\right)<+\infty$, $\lim _{n \rightarrow \infty} x_{n}=x$ and $f\left(x_{n+1}\right) \leq f\left(x_{n}\right)$ for each $n$, we have $f(x) \leq f\left(x_{n}\right)$ for each $n \in N$.

Definition 3. Let $(X, \tau)$ be a topological space and $p: X \times$ $X \rightarrow[0,+\infty]$ a function.

(1) $(X, \tau)$ is said to be $p$-complete $[33,34]$ if any sequence $\left\{x_{n}\right\}$ with $\sum_{n=1}^{\infty} p\left(x_{n}, x_{n+1}\right)<+\infty$ implies that the sequence $\left\{x_{n}\right\}$ is convergent to some $x \in X$.

(2) $(X, \tau)$ is said to be sequentially lower complete with respect to $p$, if any sequence $\left\{x_{n}\right\}$ with $\sum_{n=1}^{\infty} p\left(x_{n}, x_{n+1}\right)<+\infty$ implies that the sequence $\left\{x_{n}\right\}$ is convergent to some $x \in X$, and

$$
\liminf _{n \rightarrow \infty} p\left(x_{n}, y\right) \leq p(x, y)
$$

for any $y \in X$.

(3) Let $f: X \rightarrow(-\infty,+\infty]$ be a proper function. The topological space $(X, \tau)$ is said to be sequentially lower complete with respect to $p$ and $f$ if any sequence $\left\{x_{n}\right\}$ in $X$ satisfying $\sum_{n=1}^{\infty} p\left(x_{n}, x_{n+1}\right)<+\infty$ and $f\left(x_{n+1}\right) \leq f\left(x_{n}\right)$ for all $n$ is convergent to some $x \in X$, and (2) holds for any $y \in X$.

Remark 4. It is clearly that if $(X, \tau)$ is sequentially lower complete w.r.t. $p$, then for any proper function $f: X \rightarrow$ $(-\infty,+\infty),(X, \tau)$ is sequentially lower complete w.r.t. $p$ and $f$.

Now, we can prove the following Caristi type coincidence point theorem in the setting of topological spaces.

Theorem 5. Let $(X, \tau)$ be a topological space, $p: X \times X \rightarrow$ $[0,+\infty]$ a function, $f: X \rightarrow(-\infty,+\infty]$ a proper, bounded from below, sequentially lower monotone function with respect to $p$, and $\varphi:(-\infty,+\infty) \rightarrow(0,+\infty)$ a nondecreasing function. Assume that $(X, \tau)$ is sequentially lower complete with respect to $p$ and $f$. Let $D$ be a nonempty subset of $X$, $g: D \rightarrow X$ a surjective function, $I$ an index set, and, for each $i \in I, T_{i}: D \rightarrow 2^{X}$ a multivalued map. Then the following conclusions hold and are equivalent.

(1) Suppose that for each $x \in D$ with $g(x) \notin \bigcap_{i \in I} T_{i}(x)$, there exists $y \in X$, such that

either $0=p(g(x), y)<\varphi(f(g(x)))(f(g(x))-f(y))$,

or $\quad 0<p(g(x), y) \leq \varphi(f(g(x)))(f(g(x))-f(y))$.

Then for any $x_{0} \in X$, there exists a coincidence point $u \in D$ of $g$ and $\left\{T_{i}\right\}_{i \in I}$; that is, $g(u) \in \bigcap_{i \in I} T_{i}(u)$, such that

$$
f(g(u)) \leq f\left(x_{0}\right) .
$$

(2) Suppose that for each $x \in D$ with $g(x) \notin \bigcap_{i \in I} T_{i}(x)$, there exists an $i_{0} \in I$ and $y \in T_{i_{0}}(x)$, such that (3) holds. Then for any $x_{0} \in X$, there exists a coincidence point $u \in D$ of $g$ and $\left\{T_{i}\right\}_{i \in I}$; that is, $g(u) \in \bigcap_{i \in I} T_{i}(u)$, such that (4) holds.

Proof. (1) We take an $x_{0} \in X$, since $f \not \equiv+\infty$; without loss of generality, we can assume that $f\left(x_{0}\right)<+\infty$. Since $g: D \rightarrow X$ is a surjective function, there exists $u_{0} \in D$, such that $g\left(u_{0}\right)=x_{0}$. If $g\left(u_{0}\right) \in \bigcap_{i \in I} T_{i}\left(u_{0}\right)$, then the conclusion holds. Otherwise, by the supposition, there exists $y \in X$, such that

$$
\begin{array}{ll}
\text { either } & 0=p\left(g\left(u_{0}\right), y\right)<\varphi\left(f\left(g\left(u_{0}\right)\right)\right)\left(f\left(g\left(u_{0}\right)\right)-f(y)\right), \\
\text { or } \quad 0<p\left(g\left(u_{0}\right), y\right) \leq \varphi\left(f\left(g\left(u_{0}\right)\right)\right)\left(f\left(g\left(u_{0}\right)\right)-f(y)\right) .
\end{array}
$$

Thus,

$$
\begin{aligned}
p\left(x_{0}, y\right) & =p\left(g\left(u_{0}\right), y\right) \\
& <2 \varphi\left(f\left(g\left(u_{0}\right)\right)\right)\left(f\left(g\left(u_{0}\right)\right)-f(y)\right) \\
& =2 \varphi\left(f\left(x_{0}\right)\right)\left(f\left(x_{0}\right)-f(y)\right) .
\end{aligned}
$$

Hence,

$$
S_{1}=\left\{y \in X: p\left(x_{0}, y\right)<2 \varphi\left(f\left(x_{0}\right)\right)\left(f\left(x_{0}\right)-f(y)\right)\right\} \neq \emptyset .
$$

Obviously, for any $y \in S_{1}, f\left(x_{0}\right)>f(y)$. Thus, we can take $y_{1} \in S_{1}$ such that

$$
f\left(y_{1}\right)<\frac{1}{2}\left(f\left(x_{0}\right)+\inf _{x \in S_{1}} f(x)\right)<f\left(x_{0}\right) .
$$

Assume that $y_{n}$ has been taken, and $y_{n}=g\left(u_{n}\right)$ for $u_{n} \in D$. If $g\left(u_{n}\right) \in \bigcap_{i \in I} T_{i}\left(u_{n}\right)$, then the conclusion holds. Otherwise,

$$
S_{n+1}=\left\{y \in X: p\left(y_{n}, y\right)<2 \varphi\left(f\left(y_{n}\right)\right)\left(f\left(y_{n}\right)-f(y)\right)\right\} \neq \emptyset \text {. }
$$

Note that for any $y \in S_{n+1}$, we have $f\left(y_{n}\right)>f(y)$. Thus, we can take $y_{n+1} \in S_{n+1}$ such that

$$
\begin{gathered}
f\left(y_{n+1}\right)<\frac{1}{2}\left(f\left(y_{n}\right)+\inf _{x \in S_{n+1}} f(x)\right)<f\left(y_{n}\right), \\
p\left(y_{n}, y_{n+1}\right)<2 \varphi\left(f\left(y_{n}\right)\right)\left(f\left(y_{n}\right)-f\left(y_{n+1}\right)\right) .
\end{gathered}
$$


It remains to consider the case that there is an infinite sequence $\left\{y_{n}\right\}$ which satisfies (10) and (11). From (10), we know that $\left\{f\left(y_{n}\right)\right\}$ is a decreasing sequence. Since $\varphi$ is nondecreasing, it follows from (11) that

$$
\begin{aligned}
\sum_{n=1}^{\infty} p\left(y_{n}, y_{n+1}\right) & \leq \sum_{n=1}^{\infty} 2 \varphi\left(f\left(y_{n}\right)\right)\left(f\left(y_{n}\right)-f\left(y_{n+1}\right)\right) \\
& \leq 2 \varphi\left(f\left(x_{0}\right)\right) \sum_{n=1}^{\infty}\left(f\left(y_{n}\right)-f\left(y_{n+1}\right)\right) \\
& \leq 2 \varphi\left(f\left(x_{0}\right)\right)\left(f\left(x_{0}\right)-\inf _{x \in X} f(x)\right) \\
& <+\infty
\end{aligned}
$$

Since $(X, \tau)$ is sequentially lower complete w.r.t. $p$ and $f$, there exists $v \in X$ such that $\lim _{n \rightarrow \infty} y_{n}=v$ and $\lim _{\inf _{n \rightarrow \infty}} p\left(y_{n}, x\right) \leq p(v, x)$ for any $x \in X$. Assume that $v=g(u), u \in D$. We claim that the conclusion holds for $u$. Since $f$ is sequentially lower monotone, we have

$$
f(g(u))=f(v) \leq f\left(y_{n}\right)<f\left(x_{0}\right) .
$$

That is (4) holds. If $g(u) \notin \bigcap_{i \in I} T_{i}(u)$, then there exists $y^{\prime} \in X$, such that

$$
\begin{aligned}
p\left(v, y^{\prime}\right) & =p\left(g(u), y^{\prime}\right) \\
& <2 \varphi(f(g(u)))\left(f(g(u))-f\left(y^{\prime}\right)\right) \\
& =2 \varphi(f(v))\left(f(v)-f\left(y^{\prime}\right)\right) .
\end{aligned}
$$

It follows from $\lim \inf _{n \rightarrow \infty} p\left(y_{n}, y^{\prime}\right) \leq p\left(v, y^{\prime}\right)$ that there exists a subsequence $\left\{y_{n_{k}}\right\} \subset\left\{y_{n}\right\}$ such that

$$
\lim _{k \rightarrow \infty} p\left(y_{n_{k}}, y^{\prime}\right)=\liminf _{n \rightarrow \infty} p\left(y_{n}, y^{\prime}\right) \text {. }
$$

From this and (14) we know that there exists an $m$ such that for all $k \geq m$,

$$
p\left(y_{n_{k}}, y^{\prime}\right)<2 \varphi(f(v))\left(f(v)-f\left(y^{\prime}\right)\right) .
$$

From (13) we have

$$
p\left(y_{n_{k}}, y^{\prime}\right)<2 \varphi\left(f\left(y_{n_{k}}\right)\right)\left(f\left(y_{n_{k}}\right)-f\left(y^{\prime}\right)\right),
$$

that is, $y^{\prime} \in S_{n_{k}+1}, k \geq m$. From (10) we have

$$
2 f\left(y_{n_{k}+1}\right)-f\left(y_{n_{k}}\right) \leq \inf _{y \in S_{n_{k}+1}} f(y) \leq f\left(y^{\prime}\right) .
$$

By letting $k \rightarrow+\infty$, we get that

$$
\lim _{k \rightarrow \infty} f\left(y_{n_{k}}\right)=\lim _{n \rightarrow \infty} f\left(y_{n}\right) \leq f\left(y^{\prime}\right) .
$$

Combing with (13) we have $f(v) \leq f\left(y^{\prime}\right)$ which contradicts (14). Thus the conclusion of Theorem 5(1) holds.

(2) It is clear that Theorem $5(1) \Rightarrow$ Theorem 5(2). Now, we prove that Theorem 5(2) $\Rightarrow$ Theorem 5(1). Assume that the conditions of Theorem 5(1) are satisfied; then, for each $x \in D$, if $g(x) \notin \bigcap_{i \in I} T_{i}(x)$, there exists $y \in X$, such that (3) holds. Then we get that

$$
G(x)=\{y \in X: g(x), y \text { satisfy }(3)\} \neq \emptyset
$$

and $g(x) \notin G(x)$. For each $i \in I$, we define $\widetilde{T}_{i}: D \rightarrow 2^{X}$ by

$$
\tilde{T}_{i}(x)= \begin{cases}T_{i}(x), & \text { if } g(x) \in \bigcap_{i \in I} T_{i}(x) ; \\ T_{i}(x) \cup G(x), & \text { if } g(x) \notin \bigcap_{i \in I} T_{i}(x) .\end{cases}
$$

It is clear that $g(x) \in \bigcap_{i \in I} T_{i}(x)$ if and only if $g(x) \in$ $\bigcap_{i \in I} \widetilde{T}_{i}(x)$. Also, $\left\{\widetilde{T}_{i}\right\}$ satisfies the condition of Theorem 5(2). Then by the conclusion of Theorem 5(2), there exists a coincidence point $u \in D$ of $g$ and $\left\{\widetilde{T}_{i}\right\}_{i \in I}$; that is, $g(u) \in$ $\bigcap_{i \in I} \widetilde{T}_{i}(u)$, such that (4) holds. Therefore, there exists a coincidence point $u \in D$ of $g$ and $\left\{T_{i}\right\}_{i \in I}$; that is, $g(u) \in$ $\bigcap_{i \in I} T_{i}(u)$, such that $(4)$ holds. That is Theorem $5(1)$ holds. The proof is completed.

Remark 6. If for any $x, y \in X$ with $x \neq y$ implies that $p(x, y)>0$, then the conclusion (1) of Theorem 5 can be rewritten as: for each $x \in D$ with $g(x) \notin \bigcap_{i \in I} T_{i}(x)$ there exists $y \in X$, such that

$$
0<p(g(x), y) \leq \varphi(f(g(x)))(f(g(x))-f(y)) .
$$

In particular, if $D=X$ and $g: D \rightarrow X$ is the identity map in Theorem 5 , then we obtain the following generalized Caristi type common fixed point theorem, Caristi type fixed point theorem for set-valued, and single-valued map.

Theorem 7. Let $(X, \tau)$ be a topological space, $p: X \times X \rightarrow$ $[0,+\infty]$ a function, $f: X \rightarrow(-\infty,+\infty]$ a proper, bounded from below, sequentially lower monotone function with respect to $p$, and $\varphi:(-\infty,+\infty) \rightarrow(0,+\infty)$ a nondecreasing function. Assume that $(X, \tau)$ is sequentially lower complete w.r.t. $p$ and $f$. Then the following conclusions hold and are all equivalent to Theorem 5.

(1) Let $I$ be an index set, and, for each $i \in I$, let $T_{i}$ : $X \rightarrow 2^{X}$ be a multivalued map. Suppose further that for each $x \in X$ with $x \notin \bigcap_{i \in I} T_{i}(x)$, there exists $y \in X$, such that

$$
\begin{array}{ll}
\text { either } & 0=p(x, y)<\varphi(f(x))(f(x)-f(y)), \\
\text { or } & 0<p(x, y) \leq \varphi(f(x))(f(x)-f(y)) .
\end{array}
$$

Then for any $x_{0} \in X$, there exists a common fixed point $u \in X$ of $\left\{T_{i}\right\}_{i \in I}$; that is, $u \in \bigcap_{i \in I} T_{i}(u)$, such that

$$
f(u) \leq f\left(x_{0}\right)
$$

(2) Let $T: X \rightarrow 2^{X}$ be a multivalued map. Suppose further that for each $x \in X$ with $x \notin T(x)$, there exists $y \in X$, such that (23) holds. Then for any $x_{0} \in X, T$ has a fixed point $u \in X$, such that (24) holds. 
(3) Let $T: X \rightarrow X$ be a map. Suppose further that for each $x \in X$ with $x \neq T(x)$, there exists $y \in X$, such that (23) holds. Then for any $x_{0} \in X, T$ has a fixed point $u \in X$, such that (24) holds.

Proof. It is clear that the following implications hold: Theorem $5(1) \Rightarrow$ Theorem $7(1) \Rightarrow$ Theorem $7(2) \Rightarrow$ Theorem $7(3)$.

Now, we prove that Theorem $7(3) \Rightarrow$ Theorem 5(1). Assume that the conditions of Theorem 5 hold. It is similar to the proof of Lemma 2.1 in [36] that, by using the axiom of choice, we can prove that there exists a subset $E \subseteq D$ such that $g(E)=g(D)=X$ and $g: E \rightarrow X$ is one-to-one. Define a map $T: X \rightarrow X$ by

$$
T(x)= \begin{cases}x, & \text { if } g(u)=x \in \bigcap_{i \in I} T_{i}(u), u \in E \\ y, & \text { if } g(u)=x \notin \bigcap_{i \in I} T_{i}(u), u \in E\end{cases}
$$

where $y \in X$ such that either

$$
\begin{aligned}
0 & =p(g(u), y)=p(x, y) \\
& <\varphi(f(g(u)))(f(g(u))-f(y)) \\
& =\varphi(f(x))(f(x)-f(y)),
\end{aligned}
$$

or

$$
\begin{aligned}
0 & <p(g(u), y)=p(x, y) \\
& \leq \varphi(f(g(u)))(f(g(u))-f(y)) \\
& =\varphi(f(x))(f(x)-f(y)) .
\end{aligned}
$$

Then $T$ satisfies the condition of Theorem 7(3); thus for any $x_{0} \in X, T$ has a fixed point $v \in X$, such that $f(v) \leq f\left(x_{0}\right)$ holds. Since $g(E)=X$, there exists $u \in E \subset D$, such that $g(u)=v$. Then by the definition of $T$, we get that $g(u)=v \in$ $\bigcap_{i \in I} T_{i}(u)$. That is, the conclusion of Theorem 5 holds. The proof is completed.

The following corollary is an extension of the results in $[19,20]$. In Corollary 8 , we remove the condition that $\eta$ is nondecreasing, which is used in $[19,20]$.

Corollary 8. Let $(X, d)$ be a completed metric space. Suppose that $\eta:[0,+\infty) \rightarrow(-\infty,+\infty)$ satisfies $\eta(0)=0$ and that $f: X \rightarrow(-\infty,+\infty)$ is lower semicontinuous on $X$, and there exist $x_{0} \in X$ and two real numbers $a, b \in(-\infty,+\infty)$, such that

$$
f(x) \geq a d\left(x, x_{0}\right)+b
$$

and one of the following conditions is satisfied:

(i) $a \geq 0, \eta$ is nonnegative on $W=\{d(x, y): x, y \in X\}$, and there exist $c>0$ and $\varepsilon>0$ such that

$$
\eta(t) \geq c t, \quad \forall t \in\{t \geq 0: \eta(t) \leq \varepsilon\} \cap W
$$

(ii) $a<0, \eta(t)+$ at is nonnegative on $W$, and there exist $c>0$ and $\varepsilon>0$ such that

$\eta(t)+a t \geq c t, \quad \forall t \in\{t \geq 0: \eta(t)+a t \leq \varepsilon\} \cap W$.

Then each Caristi type mapping $T: X \rightarrow X$ (i.e., satisfying $\eta(d(x, T x)) \leq f(x)-f(T x), \forall x \in X)$ has a fixed point $v \in X$.

Proof.

Case (i). It follows from $a \geq 0$ and (28) that $f$ is a bounded from below, lower semicontinuous function on $X$. Let

$$
\alpha=\inf _{x \in X} f(x), \quad Q=\{x \in X: f(x) \leq \alpha+\varepsilon\} .
$$

Then, as in the proof of the Theorem 1 in $[20]$ that $(Q, d)$ is a complete metric space, $T Q \subset Q$, and

$$
0 \leq \eta(d(x, T x)) \leq f(x)-f(T x) \leq \varepsilon, \quad \forall x \in Q .
$$

Thus we have, by (29),

$$
c d(x, T x) \leq \eta(d(x, T x)) \leq f(x)-f(T x) \leq \varepsilon, \quad \forall x \in Q .
$$

Define a function $p: Q \times Q \rightarrow[0,+\infty]$ by $p(x, y)=$ $c d(x, y)$. Let $\left\{x_{n}\right\}$ be a sequence in $Q$, such that

$$
\sum_{n=1}^{\infty} p\left(x_{n}, x_{n+1}\right)=\sum_{n=1}^{\infty} c d\left(x_{n}, x_{n+1}\right)<+\infty .
$$

This implies that $\left\{x_{n}\right\}$ is a Cauchy sequence in $Q$. Since $(Q, d)$ is a complete metric space, $\left\{x_{n}\right\}$ is a convergent sequence in $Q$. If $\lim _{n \rightarrow \infty} x_{n}=x$, then for any $y \in Q, \lim _{n \rightarrow \infty} p\left(x_{n}, y\right)=$ $\lim _{n \rightarrow \infty} c d\left(x_{n}, y\right)=c d(x, y)=p(x, y)$. Thus, $(Q, d)$ is sequentially lower complete w.r.t. $p$. Clearly, all conditions of Theorem 7 are satisfied. Therefore, $T$ has a fixed point in $Q$.

Case (ii). Let

$$
\begin{gathered}
\psi(x)=f(x)-a d\left(x, x_{0}\right), \quad \forall x \in X, \\
\eta_{1}(t)=\eta(t)+a t, \quad \forall t \in[0,+\infty) .
\end{gathered}
$$

Then we have

$$
\eta_{1}(d(x, T x)) \leq \psi(x)-\psi(T x), \quad \forall x \in X .
$$

Thus, the conclusion can be deduced by Case (i). The proof is completed.

In Theorem 9, by using Theorem 5, we present a generalized Ekeland type variational principle, maximal element theorem for a family of multivalued maps, equilibrium theorem, and a generalized Takahashi minimization theorem in topological spaces and prove the equivalence among these results.

Theorem 9. Let $(X, \tau)$ be a topological space, $p: X \times X \rightarrow$ $[0,+\infty]$ a function, $f: X \rightarrow(-\infty,+\infty]$ a proper, bounded from below, sequentially lower monotone function with respect 
to $p$, and $\varphi:(-\infty,+\infty) \rightarrow(0,+\infty)$ a nondecreasing function. Assume that $(X, \tau)$ is sequentially lower complete with respect to $p$ and $f$. Then for any $x_{0} \in X$, the following conclusions hold, and they are equivalent to Theorem 5.

(I) (Ekeland type variational principle in topological spaces) There exists $v \in X$, such that $f(v) \leq f\left(x_{0}\right)$ and

$$
\begin{array}{r}
p(v, x)=\varphi(f(v))(f(v)-f(x)), \\
\forall x \in X \text { with } p(v, x)=0, \\
p(v, x)>\varphi(f(v))(f(v)-f(x)), \\
\forall x \in X \text { with } p(v, x)>0 .
\end{array}
$$

(II) (Maximal element for a family of multivalued maps in topological spaces) Let $I$ be any index set, and, for each $i \in I$, let $T_{i}: X \rightarrow 2^{X}$ be a multivalued map. Assume that for each $(x, i) \in X \times I$ with $T_{i}(x) \neq \emptyset$, there exists $y=y(x, i) \in X$, such that (23) holds. Then there exists $v \in X$, such that $f(v) \leq f\left(x_{0}\right)$ and $T_{i}(v)=\emptyset$ for each $i \in I$.

(III) (Equilibrium theorem in topological spaces) Let $F$ : $X \times X \rightarrow(-\infty,+\infty]$ be a proper, bounded from below, sequentially lower monotone function in the first argument. Suppose that there exists $w \in X$ such that for each $x$ with

$$
\{u \in X: F(x, u)>0\} \neq \emptyset
$$

there exists $y=y(x) \in X$ such that

$$
\begin{array}{ll}
\text { either } & 0=p(x, y)<\varphi(F(x, w))(F(x, w)-F(y, w)), \\
\text { or } & 0<p(x, y) \leq \varphi(F(x, w))(F(x, w)-F(y, w)) .
\end{array}
$$

If $(X, \tau)$ is sequentially lower complete w.r.t. $p$ and $F(\cdot, w)$, then there exists $v \in X$, such that $F(v, w) \leq$ $F\left(x_{0}, w\right)$ and $F(v, y) \leq 0$, for all $y \in X$.

(IV) (Generalized Takahashi minimization theorem in topological spaces) Suppose that for any $x$ with $f(x)>$ $\inf _{x \in X} f(x)$, there exists $y \in X$ such that (23) holds. Then there exists $v \in X$, such that $f(v) \leq f\left(x_{0}\right)$ and $f(v)=\inf _{x \in X} f(x)$.

Proof. "Theorem 5(1) $\Rightarrow$ Theorem 9(II)." If the conclusion of (II) does not hold, then for any $x \in X$, there exists $i$, such that $T_{i}(x) \neq \emptyset$. By the hypotheses of (II), there exists $y=y(x, i) \in$ $X$, such that (23) holds; thus, $y \neq x$. Let $D=X, g=I_{d}$ (the identical map of $X$ ),

$$
H_{i}(x)= \begin{cases}\left(T_{i}(x) \cup\{y(x, i)\}\right) \backslash\{x\}, & \text { if } T_{i}(x) \neq \emptyset, \\ \{x\}, & \text { if } T_{i}(x)=\emptyset .\end{cases}
$$

Then the conditions of Theorem 5(1) are satisfied for $\left\{H_{i}\right\}_{i \in I}$, $D=X$ and $g=I_{d}$. Thus, from Theorem 5(1) there exists $v \in X$ such that $v \in \bigcap_{l \in I} H_{l}(v)$. This is a contradiction with the definition of $H_{i}$. Therefore, there exists $v \in X$ with $f(v) \leq$ $f\left(x_{0}\right)$ such that $T_{i}(v)=\emptyset$ for any $i \in I$.

"(II) $\Rightarrow$ (III)" Let

$$
T_{y}(x)= \begin{cases}\{x\}, & \text { if } F(x, y)>0 \\ \emptyset, & \text { if } F(x, y) \leq 0\end{cases}
$$

From this we know that if $T_{y}(x) \neq \emptyset$, then $F(x, y)>0$. By the hypotheses of (III) there exists $z=z(x) \in X$, such that

$$
\begin{array}{ll}
\text { either } & 0=p(x, z)<\varphi(F(x, w))(F(x, w)-F(z, w)) \text {, } \\
\text { or } & 0<p(x, z) \leq \varphi(F(x, w))(F(x, w)-F(z, w))
\end{array}
$$

By using (II) for $f(x)=F(x, w)$, there exists $v \in X$, such that $F(v, w) \leq F\left(x_{0}, w\right)$ and $T_{y}(v)=\emptyset$ for any $y \in X$; that is $F(v, y) \leq 0$, for any $y \in X$.

$$
\begin{gathered}
\text { “(III) } \Rightarrow(\mathrm{IV}) " \text { Let } F(x, y)=f(x)-\inf _{x \in X} f(x) \text {. If } \\
\{u \in X: F(x, u)>0\} \neq \emptyset,
\end{gathered}
$$

then $f(x)>\inf _{x \in X} f(x)$. Fix $w \in X$; then $F(x, w) \leq F\left(x_{0}, w\right)$ if and only if $f(x) \leq f\left(x_{0}\right)$. By the hypothesis of (IV), there exists $y=y(x) \in X$, such that either

$$
\begin{aligned}
0 & =p(x, y)<\varphi(f(x))(f(x)-f(y)) \\
& =\varphi(f(x))(F(x, w)-F(y, w)),
\end{aligned}
$$

or

$$
\begin{aligned}
0 & <p(x, y) \leq \varphi(f(x))(f(x)-f(y)) \\
& =\varphi(f(x))(F(x, w)-F(y, w)) .
\end{aligned}
$$

Define $\psi:(-\infty,+\infty] \rightarrow(0,+\infty)$ by $\psi(t)=\varphi(t+$ $\left.\inf _{x \in X} f(x)\right)$. Then $\psi(F(x, y))=\varphi(f(x))$; thus, the hypotheses of (III) are satisfied for $\psi$ and $F$. It follows from (III) that there exists $v \in X$, such that $F(v, w) \leq F\left(x_{0}, w\right)$ and $F(v, y) \leq 0$, for any $y \in X$. This implies that $f(v) \leq f\left(x_{0}\right)$ and $f(v) \leq \inf _{x \in X} f(x)$, that is, $f(v)=\inf _{x \in X} f(x)$.

"(IV) $\Rightarrow$ (I)" If (I) does not hold, then for any $x \in X$ with $f(x) \leq f\left(x_{0}\right)$, there exists $y=y(x)$, such that

$$
\begin{array}{ll}
\text { either } & 0=p(x, y)<\varphi(f(x))(f(x)-f(y)), \\
\text { or } & 0<p(x, y) \leq \varphi(f(x))(f(x)-f(y)) .
\end{array}
$$

This implies that condition of (IV) holds on $X_{1}=\{x \in X$ : $\left.f(x) \leq f\left(x_{0}\right)\right\}$. Then, by (IV) there exists $v \in X_{1}$, such that $f(v) \leq f\left(x_{0}\right)$ and $f(v)=\inf _{x \in X_{1}} f(x)=\inf _{x \in X} f(x)$. This is a contradiction with (46). Thus, (I) holds.

"(I) $\Rightarrow$ Theorem 5(1)" From (I), there exists $v \in X$, such that $f(v) \leq f\left(x_{0}\right)$ and (37) holds. Since $g$ is a surjective mapping, there exists a $u \in D$, such that $g(u)=v$. We claim that $g(u) \in \bigcap_{l \in I} T_{l}(u)$. If $g(u) \notin \bigcap_{l \in I} T_{l}(u)$, by the hypotheses of Theorem 5(1), there exists $y \in X$, such that (3) holds. This is a contradiction with (37). Thus, $g(u) \in \bigcap_{l \in I} T_{l}(u)$ and $f(g(u)) \leq f\left(x_{0}\right)$. That is, Theorem 5(1) holds.

The proof is completed. 
Remark 10. Theorem 5-Theorem 9 also present Caristi type coincidence point theorem, Ekeland type variational principle, and their equivalences in $p$-complete topological spaces. Moreover, from [34] we know that $d$-complete topological spaces include $d$-complete symmetric (semimetric) spaces and complete quasimetric spaces.

\section{Applications to Some Non-Metric Spaces}

In this section, we show that our results in section two can be used with many nonmetric spaces. The reader may refer to the references $[6,13,15,25,28,37]$ for the notions and symbols in this section.

In [6], the authors introduce the concept of $Q$-function in quasimetric spaces which generalizes the notion of the $\tau$-function and $w$-distance, and they also prove an Ekeland variational principle as well as its equivalences in such spaces.

For the convenience of the reader we present the main concept of quasimetric space in the following (refer to [38]).

Let $X$ be a nonempty set. A real valued function $d: X \times$ $X \rightarrow[0,+\infty)$ is said to be a quasi-semimetric on $X$ if the following conditions are satisfied:

(QM1) $d(x, y) \geq 0$ and $d(x, x)=0$ for all $x, y \in X$;

(QM2) $d(x, y) \leq d(x, z)+d(z, y)$ for all $x, y, z \in X$.

If further

(QM3) $d(x, y)=d(y, x)=0$ implies $x=y$ for all $x, y \in X$, then $d: X \times X \rightarrow[0,+\infty)$ is said to be a quasimetric on $X$. A nonempty set $X$ together with a quasimetric $d$ (or quasi-semimetric $d$ ) is called a quasimetric space (or quasi-semimetric space), and it is denoted by $(X, d)$. If $(X, d)$ is a quasi-semimetric space, for $x \in X$ and $r>0$, we define the balls in $X$ by the formula

$B(x, r)=\{y \in X: d(x, y)<r\}$-the open ball, and

$B[x, r]=\{y \in X: d(x, y) \leq r\}$-the closed ball.

The topology $\tau$ of a quasi-semimetric $(X, d)$ can be defined starting from the family $V(x)$ of neighborhoods of an arbitrary point $x \in X$ :

$V \in V(x) \Leftrightarrow \exists r>0$ such that $B(x, r) \subset V \Leftrightarrow \exists r^{\prime}>0$ such that $B\left[x, r^{\prime}\right] \subset V$.

The convergence of a sequence $\left\{x_{n}\right\}$ to $x$ with respect to $\tau$ can be characterized by $d\left(x, x_{n}\right) \rightarrow 0$.

Definition 11. Let $(X, d)$ be a quasi-semimetric space. A sequence $\left\{x_{n}\right\}$ in $X$ is said to be

(i) left $d$-Cauchy if for each $\varepsilon>0$ there is a point $x$ in $X$ and an integer $k$ such that $d\left(x, x_{m}\right)<\varepsilon$ for all $m \geq k$;

(ii) right $d$-Cauchy if for each $\varepsilon>0$ there is a point $x$ in $X$ and an integer $k$ such that $d\left(x_{m}, x\right)<\varepsilon$ for all $m \geq k$;

(iii) $d$-Cauchy if for each $\varepsilon>0$ there is an integer $k$ such that $d\left(x_{r}, x_{s}\right)<\varepsilon$ for all $r, s \geq k$;

(iv) right $K$-Cauchy if for each $\varepsilon>0$ there is an integer $k$ such that $d\left(x_{r}, x_{s}\right)<\varepsilon$ for all $r \geq s \geq k$; (v) left $K$-Cauchy if for each $\varepsilon>0$ there is an integer $k$ such that $d\left(x_{r}, x_{s}\right)<\varepsilon$ for all $s \geq r \geq k$;

(vi) weakly left (right) $K$-Cauchy if for each $\varepsilon>0$ there is an integer $k$ such that $d\left(x_{k}, x_{m}\right)<\varepsilon\left(d\left(x_{m}, x_{k}\right)<\varepsilon\right)$ for all $m \geq k$;

(vii) corresponding to the seven definitions of Cauchy sequence in a quasi-semimetric space, we have seven notions of completeness: $X$ is said to be left (right) $d$-, [weakly] left (right) $K$-, or $d$-sequentially complete if every left (right) $d$-, [weakly] left (right) $K$-, or $d$ (resp.) Cauchy sequence in $X$ converges to some point in $X$ (with respect to the topology $\tau$ induced on $X$ by $d)$.

Remark 12. The implications between the seven notions of Cauchyness (refer to [38]) are as follows: $d$-Cauchy $\Rightarrow$ left and right $K$-Cauchy, left (right) $K$-Cauchy $\Rightarrow$ weakly left (right) $K$-Cauchy $\Rightarrow$ left (right) $d$-Cauchy.

Definition 13 (see [6]). Let $(X, d)$ be a quasi-semimetric space. A function $q: X \times X \rightarrow[0,+\infty)$ is called a $Q$-function on $X$ if the following conditions are satisfied:

(Q1) for all $x, y, z \in X, q(x, z) \leq q(x, y)+q(y, z)$;

(Q2) if $x \in X,\left\{y_{n}\right\}_{n \in N}$ is a sequence in $X$ such that it converges to a point $y$ (with respect to the quasisemimetric) and $q\left(x, y_{n}\right) \leq M$ for some $M=M(x)>$ 0 , then $q(x, y) \leq M$;

(Q3) for any $\varepsilon>0$, there exists $\delta>0$ such that $q(x, y) \leq \delta$ and $q(x, z) \leq \delta$ imply $d(y, z) \leq \varepsilon$.

Lemma 14. Let $(X, d)$ be a quasi-semimetric space with one of seven completeness defined in Definition 11(vii). If $q$ is a Qfunction on $X$, then $(X, d)$ is sequentially lower complete w.r.t. $q$.

Proof. Assume that $\left\{x_{n}\right\}$ is a sequence in $X$ and $\sum_{n=1}^{\infty} q\left(x_{n}, x_{n+1}\right)<+\infty$. Let $\lambda_{n}=\sum_{k=n+1}^{\infty} q\left(x_{n}, x_{n+1}\right)$, then we have $\lim _{n \rightarrow \infty} \lambda_{n}=0$ and for any $m>n$,

$$
q\left(x_{n}, x_{m}\right) \leq \lambda_{n}
$$

By (Q3), for any $\varepsilon>0$, there exists $\delta>0$ such that $q(x, y) \leq \delta$ and $q(x, z) \leq \delta$ imply $d(y, z) \leq \varepsilon$. For the $\delta>0$, there exists $K>0$, such that $\lambda_{n}<\delta$ for any $n \geq K$. It follows from (47) that for any $r, s \geq n>K$, we have

$$
q\left(x_{n}, x_{r}\right) \leq \lambda_{n}<\delta, \quad q\left(x_{n}, x_{s}\right) \leq \lambda_{n}<\delta .
$$

Thus (Q3) implies that $d\left(x_{r}, x_{s}\right)<\varepsilon$. That is, $\left\{x_{n}\right\}$ is a $d$ Cauchy sequence. Therefore, by Remark $12,\left\{x_{n}\right\}$ is any one of seven Cauchy sequences in Definition 11. Thus, $\left\{x_{n}\right\}$ converges to some $x \in X$. Equation (47) and (Q2) imply that $q\left(x_{n}, x\right) \leq$ $\lambda_{n}$. This shows that $\lim _{n \rightarrow \infty} q\left(x_{n}, x\right)=0$. For any $y \in X$, it follows from (Q1) that

$$
q\left(x_{n}, y\right) \leq q\left(x_{n}, x\right)+q(x, y) \text {. }
$$

Therefore

$$
\liminf _{n \rightarrow \infty} q\left(x_{n}, y\right) \leq \lim _{n \rightarrow \infty} q\left(x_{n}, x\right)+q(x, y)=q(x, y) .
$$


Thus, $(X, d)$ is sequentially lower complete w.r.t. $q$. The proof is completed.

From Lemma 14 and Theorems 5, 7, and 9, we can get the following Ekeland type variational principle and its equivalences in quasi-semimetric spaces equipped with $Q$ functions, which also generalize the results in $[6,12]$.

Theorem 15. Let $(X, d)$ be a complete quasi-semimetric space with one of seven completeness defined in Definition 11(vii) and $q: X \times X \rightarrow[0,+\infty)$ a Q-function on $X$. Let $f: X \rightarrow$ $(-\infty,+\infty]$ be a proper, bounded from below, sequentially lower monotone function and $\varphi:(-\infty,+\infty) \rightarrow(0,+\infty)$ a nondecreasing function. Let $D$ be a nonempty subset of $X, g: D \rightarrow$ $X$ a surjective function, $I$ an index set and for each $i \in I, T_{i}$ : $D \rightarrow 2^{X}$ a multivalued map. Then the following conclusions hold and are equivalent.

(1) Suppose that for each $x \in D$ with $g(x) \notin \bigcap_{i \in I} T_{i}(x)$, there exists $y \in X$, such that

either $0=q(g(x), y)<\varphi(f(g(x)))(f(g(x))-f(y))$,

or

$$
0<q(g(x), y) \leq \varphi(f(g(x)))(f(g(x))-f(y)) .
$$

Then for any $x_{0} \in X$, there exists a coincidence point $u \in D$ of $g$ and $\left\{T_{i}\right\}_{i \in I}$; that is, $g(u) \in \bigcap_{i \in I} T_{i}(u)$, such that

$$
f(g(u)) \leq f\left(x_{0}\right) .
$$

(2) Suppose that for each $x \in D$ with $g(x) \notin \bigcap_{i \in I} T_{i}(x)$, there exists an $i_{0} \in I$ and $y \in T_{i_{0}}(x)$, such that (51) holds. Then for any $x_{0} \in X$, there exists a coincidence point $u \in D$ of $g$ and $\left\{T_{i}\right\}_{i \in I}$; that is, $g(u) \in \bigcap_{i \in I} T_{i}(u)$, such that (52) holds.

(3) Let $I$ be an index set, and for each $i \in I$, let $T_{i}$ : $X \rightarrow 2^{X}$ be a multivalued map. Suppose further that for each $x \in X$ with $x \notin \bigcap_{i \in I} T_{i}(x)$, there exists $y \in X$, such that

$$
\begin{array}{ll}
\text { either } & 0=q(x, y)<\varphi(f(x))(f(x)-f(y)), \\
\text { or } \quad & 0<q(x, y) \leq \varphi(f(x))(f(x)-f(y)) .
\end{array}
$$

Then for any $x_{0} \in X$, there exists a common fixed point $u \in X$ of $\left\{T_{i}\right\}_{i \in I}$; that is, $u \in \bigcap_{i \in I} T_{i}(u)$, such that

$$
f(u) \leq f\left(x_{0}\right) .
$$

(4) (Ekeland type variational principle in quasi-semimetric spaces) For any $x_{0} \in X$, there exists $v \in X$, such that $f(v) \leq f\left(x_{0}\right)$ and

$$
\begin{array}{r}
q(v, x)=\varphi(f(v))(f(v)-f(x)), \\
\forall x \in X \text { with } q(v, x)=0, \\
q(v, x)>\varphi(f(v))(f(v)-f(x)), \\
\forall x \in X \text { with } q(v, x)>0 .
\end{array}
$$

Moreover, the rest of corresponding equivalent principles in Theorem 9 hold.
In particularly, if $(X, d)$ is a complete quasi-metric space, then from Theorem 15, we have the following results.

Theorem 16. Let $(X, d)$ be a complete quasimetric space with one of seven completeness defined in Definition 11(vii) and $q: X \times X \rightarrow[0,+\infty)$ a Q-function on $X$. Let $f: X \rightarrow$ $(-\infty,+\infty]$ be a proper, bounded from below, sequentially lower monotone function and $\varphi:(-\infty,+\infty) \rightarrow(0,+\infty)$ a nondecreasing function. Let $D$ be a nonempty subset of $X, g$ : $D \rightarrow X$ a surjective function, $I$ an index set and for each $i \in I, T_{i}: D \rightarrow 2^{X}$ a multivalued map. Then the following conclusions hold and are equivalent.

(1) Suppose that for each $x \in D$ with $g(x) \notin \bigcap_{i \in I} T_{i}(x)$, there exists $y \in X \backslash\{g(x)\}$, such that

$$
q(g(x), y) \leq \varphi(f(g(x)))(f(g(x))-f(y)) .
$$

Then for any $x_{0} \in X$, there exists a coincidence point $u \in D$ of $g$ and $\left\{T_{i}\right\}_{i \in I}$; that is, $g(u) \in \bigcap_{i \in I} T_{i}(u)$, such that

$$
f(g(u)) \leq f\left(x_{0}\right)
$$

(2) Suppose that for each $x \in D$ with $g(x) \notin \bigcap_{i \in I} T_{i}(x)$, there exists an $i_{0} \in I$ and $y \in T_{i_{0}}(x) \backslash\{g(x)\}$, such that (56) holds. Then for any $x_{0} \in X$, there exists a coincidence point $u \in D$ of $g$ and $\left\{T_{i}\right\}_{i \in I}$; that is, $g(u) \in$ $\bigcap_{i \in I} T_{i}(u)$, such that (57) holds.

(3) Let $I$ be an index set, and for each $i \in I$, let $T_{i}: X \rightarrow$ $2^{X}$ be a multivalued map. Suppose further that for each $x \in X$ with $x \notin \bigcap_{i \in I} T_{i}(x)$, there exists $y \in X \backslash\{x\}$, such that

$$
q(x, y) \leq \varphi(f(x))(f(x)-f(y)) .
$$

Then for any $x_{0} \in X$, there exists a common fixed point $u \in X$ of $\left\{T_{i}\right\}_{i \in I}$; that is, $u \in \bigcap_{i \in I} T_{i}(u)$, such that

$$
f(u) \leq f\left(x_{0}\right)
$$

(4) (Ekeland type variational principle in quasimetric spaces) For any $x_{0} \in X$, there exists $v \in X$, such that $f(v) \leq f\left(x_{0}\right)$ and

$$
q(v, x)>\varphi(f(v))(f(v)-f(x)), \quad \forall x \in X \text { with } x \neq v .
$$

Proof. The equivalence of the conclusions (1)-(4) is clear. We only prove (4). It follows from (4) of Theorem 15 that for any $x_{0} \in X$, there exists $v \in X$, such that $f(v) \leq f\left(x_{0}\right)$ and

$$
\begin{array}{r}
q(v, x)=\varphi(f(v))(f(v)-f(x)), \\
\forall x \in X \text { with } q(v, x)=0, \\
q(v, x)>\varphi(f(v))(f(v)-f(x)), \\
\forall x \in X \text { with } q(v, x)>0 .
\end{array}
$$


If $v$ satisfies (60), then conclusion (4) is proved. Otherwise, there exists $x \in X \backslash\{v\}$, such that

$$
0=q(v, x)=\varphi(f(v))(f(v)-f(x)) .
$$

If $x$ and $y$ satisfy (62), then $q(v, x)=0$ and $q(v, y)=0$. By using (Q3) in Definition 13, we get that $d(x, y)=0$. It follows from (QM1) that $d(x, x)=0$, and then (QM3) implies that $x=y$. That is, there is only one point $\bar{x}$ which satisfies (62). Let

$$
S(x)=\{y \in X: q(x, y) \leq \varphi(f(x))(f(x)-f(y))\} .
$$

Then $S(v)=\{\bar{x}\}$. Since $\bar{x} \in S(v)$, we can imply that $S(\bar{x}) \subset$ $S(v)=\{\bar{x}\}$. This shows that for any $x \neq \bar{x}, x \notin S(\bar{x})$; that is, $\bar{x}$ satisfies (60). The proof is completed.

Definition 17 (see, e.g., [37]). Let $X$ be a real vector space; a collection $\mathscr{B}$ of subsets of $X$ is called a vector bornology on $X$, if it satisfies the following conditions:

(B1) $x \in X$ implies that $\{x\} \in \mathscr{B}$;

(B2) $B_{1} \subset B_{2}$ and $B_{2} \in \mathscr{B}$ imply that $B_{1} \in \mathscr{B}$;

(B3) $B_{1}, B_{2} \in \mathscr{B}$ implies that $B_{1} \cup B_{2} \in \mathscr{B}$;

(B4) $B_{1}, B_{2} \in \mathscr{B}$ implies that

$$
B_{1}+B_{2}=\left\{x+y: x \in B_{1}, y \in B_{2}\right\} \in \mathscr{B} ;
$$

(B5) for any bounded interval $I \subset(-\infty,+\infty), B \in \mathscr{B}$ implies that

$$
I \cdot B=\{\alpha x: \alpha \in I, x \in B\} \in \mathscr{B} .
$$

In view of (B5), if $B \in \mathscr{B}$, so is its balanced hull $B_{b}$ which is defined by $B_{b}=[-1,1] \cdot B$.

Definition 18. The ordered pair $(X, \mathscr{B})$ is called a bornological vector space (in short: BVS), and every element of $\mathscr{B}$ is called a bounded subset (with respect to $\mathscr{B}$ ).

Definition 19 (see, e.g., $[28,37])$. Let $(X, \mathscr{B})$ be a bornological vector space.

(i) A sequence $\left\{x_{n}\right\}$ in $X$ is said to be Mackeyconvergent (or $M$-convergent) to a point $x$, denoted by $\lim _{n \rightarrow \infty}^{b} x_{n}=x$, if there is a balanced $B \in \mathscr{B}$ and a sequence of positive real numbers $\left\{\lambda_{n}\right\}$ such that $\lim _{n \rightarrow \infty} \lambda_{n}=0$ and $x_{n}-x \in \lambda_{n} B$ for any $n \in N$. Also, we say that $x$ is a bornological limit of $\left\{x_{n}\right\}$.

(ii) A sequence $\left\{x_{n}\right\}$ in $X$ is said to be Mackey-Cauchy (or $M$-Cauchy) if there is a balanced $B \in \mathscr{B}$ and a double sequence of positive real numbers $\left\{\lambda_{m n}\right\}$ such that $\lim _{m, n \rightarrow \infty} \lambda_{m n}=0$ and $x_{m}-x_{n} \in \lambda_{m n} B$ for any $m, n \in N$.

(iii) $A \subset X$ is said to be Mackey-closed (or $M$-closed) if it contains all bornological limits of any sequences in A.

(iv) $A \subset X$ is said to be Mackey-complete (or $M$ complete) if every $M$-Cauchy sequence in $A$ will be $M$-convergent to some element in $A$. (v) A BVS $(X, \mathscr{B})$ is said to be separated if every $M$ convergent sequence is $M$-convergent to exactly one bornological limit.

Remark 20. From Lemma 2.13 in [28] we know that if $A \subset X$ is a $M$-complete subset, then $A$ is $M$-closed. On the other hand, if $(X, \mathscr{B})$ is $M$-complete and $A \subset X$ is $M$-closed, then $A$ is $M$-complete. For the details about BVS, one can refer to $[17,28,37]$.

The collection of all (complements of) $M$-closed subsets of $X$ defines a topology on $X$, and we called it bornological topology. Therefore, $(X, \mathscr{B})$ endowed this topology is a topological space (but, from Remark 2.4 in [28], we can see that it is rarely a vector topology with respect to the algebraic structure of $X)$. In the following, we will assume that $(X, \mathscr{B})$ is separated; that is (v) in Definition 19 holds.

Let $X$ be a separated bornological vector space and $P: X \rightarrow(-\infty,+\infty]$ a positively homogeneous subadditive function. By Lemma 4.4 in [28], $P(x)>0$ for any nonzero $x$ if $P$ satisfies the following condition:

(P1) the set $C=\{x \in X: P(x) \leq 1\}$ is $M$-complete and bounded.

Lemma 21. Let $p: X \times X \rightarrow(-\infty,+\infty)$ be defined by $p(x, y)=P(x-y)$. If $(P 1)$ holds, then $(X, \mathscr{B})$ is sequentially lower complete with respect to $p$.

Proof. Let $\left\{x_{n}\right\}$ be a sequence in $X$ and $\sum_{n=1}^{\infty} p\left(x_{n}, x_{n+1}\right)=$ $\sum_{n=1}^{\infty} P\left(x_{n}-x_{n+1}\right)<+\infty$. Then for any $0<\delta<1$, there exists a positive integer $n_{0}$, such that $\sum_{n=n_{0}}^{\infty} P\left(x_{n}-x_{n+1}\right)<\delta$. Since $P$ is subadditive, we get that for any $m>n_{0}$,

$$
P\left(x_{n_{0}}-x_{m}\right) \leq \sum_{n=n_{0}}^{m-1} P\left(x_{n}-x_{n+1}\right)<\delta .
$$

From this we have

$$
P\left(x_{n_{0}}-x_{m}\right) \leq \delta<1 .
$$

So we have $x_{n_{0}}-x_{m} \in C$. For any $m>n$, let $\lambda_{m n}=$ $\sum_{k=n}^{m-1} P\left(x_{k}-x_{k+1}\right)$, and then we have $P\left(x_{n}-x_{m}\right) \leq \lambda_{m n}$, and $\lim _{m, n \rightarrow \infty} \lambda_{m n}=0$. It follows from

$$
P\left(\left(x_{n_{0}}-x_{m}\right)-\left(x_{n_{0}}-x_{n}\right)\right)=P\left(x_{n}-x_{m}\right) \leq \lambda_{m n}
$$

that $\left(x_{n_{0}}-x_{m}\right)-\left(x_{n_{0}}-x_{n}\right) \in \lambda_{m n} C \subset \lambda_{m n} C_{b}$ where $C_{b}$, is the (bounded) balanced hull of $C$. That is, $\left\{x_{n_{0}}-x_{n}\right\}$ is $M$-Cauchy. Since $C$ is $M$-complete, $\left\{x_{n_{0}}-x_{n}\right\}$ is $M$-convergent. Thus, $\left\{x_{n}\right\}$ is also $M$-convergent. Assume that $\left\{x_{n}\right\}$ is $M$-convergent to a point $x$. If we set

$$
\lambda_{n}=\sum_{k=n}^{\infty} P\left(x_{k}-x_{k+1}\right),
$$

then $\lambda_{m n} \leq \lambda_{n}$, and hence $\lambda_{m n} C \subset \lambda_{n} C$ whenever $m>n$. It follows from (68) that $x_{n}-x_{m} \in C$ for $m>n$. Since $\lambda_{n} C$ is $M$-closed by (P1), we have

$$
\lim _{m \rightarrow \infty}^{b}\left(x_{n}-x_{m}\right)=x_{n}-x \in \lambda_{n} C .
$$


Consequently, $P\left(x_{n}-x\right) \leq \lambda_{n}$, and hence $\lim _{n \rightarrow \infty} P\left(x_{n}-x\right)=$ 0 . Since $P\left(x_{n}-y\right) \leq P\left(x_{n}-x\right)+P(x-y)$, we have

$$
\begin{aligned}
\liminf _{n \rightarrow \infty} P\left(x_{n}-y\right) & \leq \lim _{n \rightarrow \infty} P\left(x_{n}-x\right)+P(x-y) \\
& =P(x-y) .
\end{aligned}
$$

That is,

$$
\liminf _{n \rightarrow \infty} p\left(x_{n}, y\right) \leq p(x, y)
$$

Thus, $(X, \mathscr{B})$ is sequentially lower complete with respect to $p$. The proof is completed.

From Lemma 21 and Theorem 9, we can get the following Ekeland type variational principle in bornological vector space, which is also proved in $[17,28]$.

Theorem 22 (Ekeland type variational principle in bornological vector space). Let $(X, \mathscr{B})$ be a separated bornological vector space and $P: X \rightarrow(-\infty,+\infty]$ a positively homogeneous subadditive function satisfying the condition (P1). Let $f: X \rightarrow(-\infty,+\infty]$ be a proper, bounded from below, sequentially lower monotone function and $\varphi$ : $(-\infty,+\infty) \rightarrow(0,+\infty)$ a nondecreasing function. Then for any $x_{0} \in X$, there exists $v \in X$, such that $f(v) \leq f\left(x_{0}\right)$ and

$$
P(v-x)>\varphi(f(v))(f(v)-f(x)), \quad \forall x \neq v .
$$

Moreover, the corresponding equivalent principles in Theorem 9 hold.

Definition 23 (see [25]). Let $X$ be a uniform space. An extended real-valued function $p: X \times X \rightarrow[0,+\infty]$ is called a $q$-distance on $X$ if the following conditions are satisfied:

(q1) for any $x, y, z \in X, p(x, z) \leq p(x, y)+p(y, z)$;

(q2) every sequence $\left\{y_{n}\right\} \subset X$ with $p\left(y_{n}, y_{m}\right) \rightarrow 0(m>$ $n \rightarrow \infty)$ is a Cauchy sequence and in the case $p\left(y_{n}, y\right) \rightarrow 0$ implies that $y_{n} \rightarrow y$ in $X$;

(q3) for $x, y, z \in X, p(z, x)=0$ and $p(z, y)=0$ imply $x=y$.

Here $p\left(y_{n}, y_{m}\right) \rightarrow 0(m>n \rightarrow \infty)$ means that for any $\varepsilon>0$, there exists $n_{0} \in N$ such that $p\left(y_{n}, y_{m}\right)<\varepsilon$ for all $m>n \geq n_{0}$.

Definition 24 (see [25]). Let $(X, \mathcal{U})$ be a uniform space and $p$ a $q$-distance on $X$. A proper function $f: X \rightarrow(-\infty,+\infty]$ is said to be sequentially lower monotone with respect to $p$ (in short, sequentially lower monotone with respect to $p$ ) if for any sequence $\left\{x_{n}\right\}$ in $X$ satisfying $p\left(x_{n}, x_{m}\right) \rightarrow 0(m>n \rightarrow$ $\infty), p\left(x_{n}, \bar{x}\right) \rightarrow 0(n \rightarrow \infty)$ and $f\left(x_{n+1}\right) \leq f\left(x_{n}\right)$, we have $f(\bar{x}) \leq f\left(x_{n}\right)$ for each $n \in N$.

Definition 25 (see [25]). Let $(X, \mathcal{U})$ be a uniform space, $p$ a $q$-distance on $X$, and $f: X \rightarrow(-\infty,+\infty)$ a proper function. $(X, \mathcal{U})$ is said to be sequentially complete with respect to $(p, f \downarrow)$ if for any sequence $\left\{x_{n}\right\}$ in $X$ satisfying $p\left(x_{n}, x_{m}\right) \rightarrow$ $0(m>n \rightarrow \infty)$ and $f\left(x_{n+1}\right) \leq f\left(x_{n}\right)$ for each $n \in N$, there exists $\bar{x} \in X$ such that $p\left(x_{n}, \bar{x}\right) \rightarrow 0(n \rightarrow \infty)$.
Lemma 26. Let $(X, \mathcal{U})$ be a uniform space, $p$ a q-distance on $X$, and $f: X \rightarrow(-\infty,+\infty]$ a sequentially lower monotone with respect to $p$, proper function, bounded from below. If $(X, \mathcal{U})$ is sequentially lower complete with respect to $(p, f \downarrow)$, then $(X, \mathcal{U})$ is sequentially lower complete with respect to $p$ and $f$.

Proof. Assume that $\left\{x_{n}\right\}$ is a sequence in $X$ with $f\left(x_{n+1}\right) \leq$ $f\left(x_{n}\right)$ for each $n \in N$ and $\sum_{n=1}^{\infty} p\left(x_{n}, x_{n+1}\right)<+\infty$. Let $\lambda_{n}=$ $\sum_{k=n+1}^{\infty} p\left(x_{n}, x_{n+1}\right)$, then we have that $\lim _{n \rightarrow \infty} \lambda_{n}=0$, and for any $m>n$,

$$
p\left(x_{n}, x_{m}\right) \leq \lambda_{n}
$$

This shows that $p\left(x_{n}, x_{m}\right) \rightarrow 0(m>n \rightarrow \infty)$. By using Definition 23 and Definition 25, we get that there exists $\bar{x} \in X$ such that $p\left(x_{n}, \bar{x}\right) \rightarrow 0(n \rightarrow \infty)$ and $\left\{x_{n}\right\}$ converges to $\bar{x}$. Then for any $y \in X$, by $p\left(x_{n}, y\right) \leq p\left(x_{n}, \bar{x}\right)+p(\bar{x}, y)$ we have that

$$
\liminf _{n \rightarrow \infty} p\left(x_{n}, y\right) \leq \lim _{n \rightarrow \infty} p\left(x_{n}, \bar{x}\right)+p(\bar{x}, y)=p(\bar{x}, y) .
$$

Thus, $(X, \mathcal{U})$ is sequentially lower complete w.r.t. $p$ and $f$. The proof is completed.

From Theorems 5, 7, and 9, we can get the following Caristi type coincidence point theorem and Ekeland type variational principle in uniform space equipped with $q^{-}$ distance. From Lemma 26, we also see that this is a transformation of the results appeared in [25].

Theorem 27. Let $(X, \mathcal{U})$ be a uniform space, $p$ a q-distance on $X$, and $f: X \rightarrow(-\infty,+\infty]$ a sequentially lower monotone with respect to $p$, proper function, bounded from below. Let $D$ be a nonempty subset of $X, g: D \rightarrow X$ a surjective function, $I$ an index set, and, for each $i \in I, T_{i}: D \rightarrow 2^{X}$ a multivalued map. If $(X, \mathcal{U})$ is sequentially complete with repect to $p$ and $f$ and $\varphi:(-\infty,+\infty) \rightarrow(0,+\infty)$ is a nondecreasing function, then the following conclusions hold and are equivalent.

(1) Suppose that for each $x \in D$ with $g(x) \notin \bigcap_{i \in I} T_{i}(x)$, there exists $y \in X \backslash\{g(x)\}$, such that

$$
p(g(x), y) \leq \varphi(f(g(x)))(f(g(x))-f(y)) .
$$

Then for any $x_{0} \in X$, there exists a coincidence point $u \in D$ of $g$ and $\left\{T_{i}\right\}_{i \in I}$; that is, $g(u) \in \bigcap_{i \in I} T_{i}(u)$, such that

$$
f(g(u)) \leq f\left(x_{0}\right)
$$

(2) Suppose that for each $x \in D$ with $g(x) \notin \bigcap_{i \in I} T_{i}(x)$, there exists an $i_{0} \in I$ and $y \in T_{i_{0}}(x) \backslash\{g(x)\}$, such that (76) holds. Then for any $x_{0} \in X$, there exists a coincidence point $u \in D$ of $g$ and $\left\{T_{i}\right\}_{i \in I}$; that is, $g(u) \in$ $\bigcap_{i \in I} T_{i}(u)$, such that (77) holds.

(3) Let $I$ be an index set, and, for each $i \in I$, let $T_{i}: X \rightarrow$ $2^{X}$ be a multivalued map. Suppose further that for 
each $x \in X$ with $x \notin \bigcap_{i \in I} T_{i}(x)$, there exists $y \in X \backslash\{x\}$, such that

$$
p(x, y) \leq \varphi(f(x))(f(x)-f(y)) .
$$

Then for any $x_{0} \in X$, there exists a common fixed point $u \in X$ of $\left\{T_{i}\right\}_{i \in I}$; that is, $u \in \bigcap_{i \in I} T_{i}(u)$, such that

$$
f(u) \leq f\left(x_{0}\right) .
$$

(4) (Ekeland type variational principle in uniform spaces) For any $x_{0} \in X$, there exists $v \in X$, such that $f(v) \leq$ $f\left(x_{0}\right)$ and

$$
p(v, x)>\varphi(f(v))(f(v)-f(x)), \quad \forall x \in X \text { with } x \neq v .
$$

Moreover, the rest of corresponding equivalent principles in Theorem 9 hold.

Proof. The equivalence of the conclusions (1)-(4) is clear. We only need to prove (4). The proof of (4) is similar to the proof of Theorem 16, by using (q3). So we delete the detail of the proof.

Remark 28. If $\varphi:(-\infty,+\infty) \rightarrow(0,+\infty)$ is upper semicontinuous and (76) is replaced by

$$
\begin{aligned}
& p(g(x), y) \\
& \quad \leq \max \{\varphi(f(g(x))), \varphi(f(y))\} \cdot(f(g(x))-f(y)),
\end{aligned}
$$

then the conclusions of Theorem 27 hold. In this case, the proof is similar to the proof of Theorem 2.1 in [11].

As noted in Remark 5.1 in [25], from our Theorem 27, we can deduce [13, Theorems 3.1 and 3.2, and Corollary 3.3] and [15, Theorems 4-6]. Furthermore, we will show that Theorem 27 improves some coincidence point theorems and their equivalences in $F$-type separated topological space (or equivalently, generating spaces of quasimetric family) and fuzzy metric spaces, which were proved in [8-11].

In the following, we will assume that $(X, \mathfrak{J})$ is an $F$ type separated topological space (or equivalently, a uniform space, see $[13,15]$ ) whose topology is generated by a separated family $\left\{q_{\lambda}\right\}_{\lambda \in \Lambda}$ of quasimetrics, where $(\Lambda, \prec)$ is a directed set. Moreover, let $\alpha: \Lambda \rightarrow(0,+\infty)$ be a nondecreasing function; that is, $\lambda, \mu \in \Lambda, \lambda \prec \mu$ implies $\alpha(\lambda) \leq \alpha(\mu)$. An extended realvalued function $p: X \times X \rightarrow[0,+\infty]$ is defined as follows:

$$
p(x, y)=\sup _{\lambda \in \Lambda} \alpha(\lambda) q_{\lambda}(x, y), \quad \forall(x, y) \in X \times X .
$$

Lemma 29. Let $(X, \mathfrak{J})$ be an F-type separated topological space (or equivalently, a uniform space) whose topology is generated by a family $\left\{q_{\lambda}\right\}_{\lambda \in \Lambda}$ of quasimetrics, and let $p$ : $X \times X \rightarrow[0,+\infty]$ be defined by $(82)$. If $(X, \Im)$ is sequentially complete, then $(X, \Im)$ is sequentially lower complete w.r.t. $p$.
Proof. Assume that a sequence $\left\{x_{n}\right\}$ in $X$ satisfies $\sum_{n=1}^{\infty} p\left(x_{n}, x_{n+1}\right)<+\infty$. This implies that the sequence $\left\{x_{n}\right\}$ satisfies $p\left(x_{n}, x_{m}\right) \rightarrow 0(m>n \rightarrow \infty)$. It follows from Examples 2.3 and 3.1 in [25] that $p$ is a $q$-distance on $X$, and there exists an $x \in X$, such that $\left\{x_{n}\right\}$ converges to $x$ and $p\left(x_{n}, x\right) \rightarrow 0$. Then for any $y \in X$,

$$
\liminf _{n \rightarrow \infty} p\left(x_{n}, y\right) \leq \liminf _{n \rightarrow \infty}\left[p\left(x_{n}, x\right)+p(x, y)\right]=p(x, y) \text {. }
$$

Thus, $(X, \mathfrak{I})$ is sequentially lower complete w.r.t. $p$. The proof is completed.

By using Lemma 29 and Theorem 27, we have the following results.

Theorem 30. Let $(X, \Im)$ be a sequentially complete and separated F-type topological space (or equivalently, a uniform space) whose topology is generated by a family $\left\{q_{\lambda}\right\}_{\lambda \in \Lambda}$ of quasimetrics, $\varphi:(-\infty,+\infty) \rightarrow(0,+\infty)$ a nondecreasing function, and $f: X \rightarrow(-\infty,+\infty]$ a proper, bounded from below, sequentially lower monotone function. Let $D$ be a nonempty subset of $X, g: D \rightarrow X$ a surjective function, and I an index set, and, for each $i \in I$, let $T_{i}: D \rightarrow 2^{X}$ be a multivalued map. Then the following conclusions hold and are equivalent.

(1) Suppose that for each $x \in D$ with $g(x) \notin \bigcap_{i \in I} T_{i}(x)$, there exists $y \in X \backslash\{g(x)\}$, such that

$$
\begin{array}{r}
\alpha(\lambda) q_{\lambda}(g(x), y) \leq \varphi(f(g(x)))(f(g(x))-f(y)), \\
\forall \lambda \in \Lambda .
\end{array}
$$

Then for any $x_{0} \in X$, there exists a coincidence point $u \in D$ of $g$ and $\left\{T_{i}\right\}_{i \in I}$; that is, $g(u) \in \bigcap_{i \in I} T_{i}(u)$, such that

$$
f(g(u)) \leq f\left(x_{0}\right) .
$$

(2) Suppose that for each $x \in D$ with $g(x) \notin \bigcap_{i \in I} T_{i}(x)$, there exists an $i_{0} \in I$ and $y \in T_{i_{0}}(x) \backslash\{g(x)\}$, such that (82) holds. Then for any $x_{0} \in X$, there exists a coincidence point $u \in D$ of $g$ and $\left\{T_{i}\right\}_{i \in I}$; that is, $g(u) \in$ $\bigcap_{i \in I} T_{i}(u)$, such that (84) holds.

(3) Let I be an index set and, for each $i \in I, T_{i}: X \rightarrow 2^{X} a$ multivalued map. Suppose further that for each $x \in X$ with $x \notin \bigcap_{i \in I} T_{i}(x)$, there exists $y \in X \backslash\{x\}$, such that

$\alpha(\lambda) q_{\lambda}(x, y) \leq \varphi(f(x))(f(x)-f(y)), \quad \forall \lambda \in \Lambda$.

Then for any $x_{0} \in X$, there exists a common fixed point $u \in X$ of $\left\{T_{i}\right\}_{i \in I}$; that is, $u \in \bigcap_{i \in I} T_{i}(u)$, such that

$$
f(u) \leq f\left(x_{0}\right) .
$$

(4) (Ekeland type variational principle in F-type topological spaces) For any $x_{0} \in X$, there exists $v \in X$, such that $f(v) \leq f\left(x_{0}\right)$ and for any $x \in X$ with $v \neq x$, there exists $\lambda_{0} \in \Lambda$, such that

$$
\alpha\left(\lambda_{0}\right) q_{\lambda_{0}}(v, x)>\varphi(f(v))(f(v)-f(x)) .
$$


The following version of coincidence point theorem is an improvement for the coincidence point theorems proved in $[8,9]$.

Theorem 31. Let $\left(X, d_{\lambda}: \lambda \in \Lambda\right)$ and $\left(Y, \delta_{\lambda}: \lambda \in \Lambda\right)$ be two sequentially complete and separated generating spaces of quasimetric family, $\alpha: \Lambda \rightarrow(0,+\infty)$ a nondecreasing function, $D$ a nonempty subset of $X, g: D \rightarrow X$ a surjective function, $h: X \rightarrow Y$ a closed mapping, $f: h(X) \rightarrow(-\infty,+\infty]$ a proper, bounded from below, sequentially lower monotone function, and $\varphi:(-\infty,+\infty) \rightarrow(0,+\infty)$ a non-decreasing function. Let $I$ be an index set, and, for each $i \in I$, let $T_{i}: D \rightarrow$ $2^{X}$ be a multivalued map. Suppose further that for each $x \in D$ with $g(x) \notin \bigcap_{i \in I} T_{i}(x)$, there exists $y \in X \backslash\{g(x)\}$, such that

$$
\begin{aligned}
& \alpha(\lambda) \max \left\{d_{\lambda}(g(x), y), c \delta_{\lambda}(h(g(x)), h(y))\right\} \\
& \quad \leq \varphi(f(h(g(x))))(f(h(g(x)))-f(h(y))), \quad \forall \lambda \in \Lambda,
\end{aligned}
$$

where $c>0$ is a given constant. Then for any $x_{0} \in X$, there exists a coincidence point $u \in D$ of $g$ and $\left\{T_{i}\right\}_{i \in I}$; that is, $g(u) \in$ $\bigcap_{i \in I} T_{i}(u)$, such that

$$
f(h(g(u))) \leq f\left(h\left(x_{0}\right)\right) .
$$

Proof. For each $\lambda \in \Lambda$, we define $q_{\lambda}: X \times X \rightarrow R$ by

$$
q_{\lambda}(x, y)=\max \left\{d_{\lambda}(x, y), c \delta_{\lambda}(h(x), h(y))\right\},
$$

and then, by Definition 2 in [15], we can verify that the collection $\left\{q_{\lambda}\right\}_{\lambda \in \Lambda}$ defined by (91) is a family of quasimetrics on $X$. Since $\left(X, d_{\lambda}: \lambda \in \Lambda\right)$ and $\left(Y, \delta_{\lambda}: \lambda \in \Lambda\right)$ are sequentially complete generating spaces of quasimetric family and $h$ is a closed mapping, we can deduce that $\left(X, q_{\lambda}\right.$ : $\lambda \in \Lambda$ ) is also a sequentially complete generating space of quasimetric family. Next, we assume that $\left\{x_{n}\right\} \subset X$ is a sequence which converges to $x$ in $\left(X, q_{\lambda}: \lambda \in \Lambda\right)$ and satisfies

$$
f\left(h\left(x_{1}\right)\right) \geq f\left(h\left(x_{2}\right)\right) \geq \cdots \geq f\left(h\left(x_{n}\right)\right) \geq \cdots .
$$

By (91) we know that $\left\{x_{n}\right\}$ converges to $x$ in $\left(X, d_{\lambda}: \lambda \in \Lambda\right)$ and $\left\{h\left(x_{n}\right)\right\}$ converges to $h(x)$ in $\left(Y, \delta_{\lambda}: \lambda \in \Lambda\right)$. Since $f: h(X) \rightarrow(-\infty,+\infty]$ is a sequentially lower monotone function, we have $f(h(x)) \leq f\left(h\left(x_{n}\right)\right)$, for each $n$, that is, $f \circ h$ is a sequentially lower monotone function on $\left(X, q_{\lambda}: \lambda \in \Lambda\right)$. Then by using Theorem 27 (1) for $\left(X, q_{\lambda}: \lambda \in \Lambda\right)$ and $f \circ h$, we can get the conclusion of Theorem 31. The proof is completed.

The following version of coincidence point theorem is an improvement for the coincidence point theorems proved in [11].

Theorem 32. Let $\left(X, d_{\lambda}: \lambda \in \Lambda\right)$ and $\left(Y, \delta_{\lambda}: \lambda \in \Lambda\right)$ be two sequentially complete generating spaces of quasimetric family, $\alpha: \Lambda \rightarrow(0,+\infty)$ a nondecreasing function, $D$ a non-empty subset of $X, g: D \rightarrow X$ a surjective function, $h: X \rightarrow Y a$ closed mapping, $f: h(X) \rightarrow(-\infty,+\infty]$ a proper, bounded from below, sequentially lower monotone function, and $\varphi$ : $(-\infty,+\infty) \rightarrow(0,+\infty)$ an upper semicontinuous function.
Let $I$ be an index set, and, for each $i \in I$, let $T_{i}: D \rightarrow 2^{X}$ be a multivalued map. Suppose further that for each $x \in D$ with $g(x) \notin \bigcap_{i \in I} T_{i}(x)$, there exists $y \in X \backslash\{g(x)\}$, such that

$$
\begin{aligned}
\alpha(\lambda) \max \left\{d_{\lambda}(g(x), y), c \delta_{\lambda}(h(g(x)), h(y))\right\} \\
\leq \max \{\varphi(f(h(g(x)))), \varphi(f(h(y)))\} \\
\times(f(h(g(x)))-f(h(y))), \quad \forall \lambda \in \Lambda,
\end{aligned}
$$

where $c>0$ is a given constant. Then for any $x_{0} \in X$, there exists a coincidence point $u \in D$ of $g$ and $\left\{T_{i}\right\}_{i \in I}$; that is, $g(u) \in$ $\bigcap_{i \in I} T_{i}(u)$, such that

$$
f(h(g(u))) \leq f\left(h\left(x_{0}\right)\right) .
$$

Proof. It follows from the proof of Theorem 16 that $f \circ h$ is a sequentially lower monotone function on $\left(X, q_{\lambda}: \lambda \in \Lambda\right)$, where $\left\{q_{\lambda}\right\}_{\lambda \in \Lambda}$ is defined by (91). Then by using Remark 28 for $\left(X, q_{\lambda}: \lambda \in \Lambda\right)$ and $f \circ h$, we can get the conclusion of Theorem 32. The proof is completed.

Remark 33. Similarly, under the conditions of Theorem 31 or Theorem 32, we can get corresponding common fixed point theorems, Ekeland's variational principle, and other equivalences of Theorems 31 and 32. On the other hand, a fuzzy metric space in the sense of Kaleva and Seikkala [39] is a generating space of quasimetric family (see, e.g., $[8,9,11])$; thus, by using our results in this section we can get coincidence point theorems and its equivalences in fuzzy metric spaces. Therefore, our results are also unified improvements of the results in [8-11].

Let $(X, \mathcal{U})$ be a uniform space, $q: X \times X \rightarrow[0,+\infty)$ a $q$ distance on $X$, and $h:[0,+\infty) \rightarrow[0,+\infty)$ a nondecreasing function, such that

$$
\int_{0}^{+\infty} \frac{d r}{1+h(r)}=+\infty
$$

and $x_{0}$ is a given point in $X$. Let $p: X \times X \rightarrow[0,+\infty)$ be defined by

$$
p(x, y)=\int_{q\left(x_{0}, x\right)}^{q\left(x_{0}, x\right)+q(x, y)} \frac{d r}{1+h(r)} .
$$

Lemma 34. Let $(X, \mathcal{U})$ be a uniform space, $q$ a $q$-distance on $X, h:[0,+\infty) \rightarrow[0,+\infty)$ a nondecreasing function satisfying (95), and $f: X \rightarrow(-\infty,+\infty]$ a sequentially lower monotone with respect to $q$, proper function, bounded from below. If $(X, \mathcal{U})$ is sequentially lower complete with respect to $(q, f \downarrow)$, then $(X, \mathcal{U})$ is sequentially lower complete with respect to $p$ and $f$, where $p$ is defined by (96).

Proof. Let $\left\{x_{n}\right\}$ be a sequence in $X$ with $f\left(x_{n+1}\right) \leq f\left(x_{n}\right)$ for each $n \in N$ and satisfy

$$
\sum_{n=1}^{\infty} p\left(x_{n}, x_{n+1}\right)=\sum_{n=1}^{\infty} \int_{q\left(x_{0}, x_{n}\right)}^{q\left(x_{0}, x_{n}\right)+q\left(x_{n}, x_{n+1}\right)} \frac{d r}{1+h(r)}<+\infty .
$$


For any $n>1$, by noting that $h:[0,+\infty) \rightarrow[0,+\infty)$ is nondecreasing, we have

$$
\begin{aligned}
\int_{q\left(x_{0}, x_{1}\right)}^{\sum_{k=0}^{n-1} q\left(x_{k}, x_{k+1}\right)} \frac{d r}{1+h(r)} \\
\quad=\int_{q\left(x_{0}, x_{1}\right)}^{q\left(x_{0}, x_{1}\right)+q\left(x_{1}, x_{2}\right)} \frac{d r}{1+h(r)}+\cdots \\
\quad+\int_{q\left(x_{0}, x_{1}\right)+\cdots+q\left(x_{n-2}, x_{n-1}\right)}^{q\left(x_{0}, x_{1}\right)+\cdots+q\left(x_{n-1}, x_{n}\right)} \frac{d r}{1+h(r)} \\
\leq \int_{q\left(x_{0}, x_{1}\right)}^{q\left(x_{0}, x_{1}\right)+q\left(x_{1}, x_{2}\right)} \frac{d r}{1+h(r)}+\cdots \\
\quad+\int_{q\left(x_{0}, x_{n}\right)}^{q\left(x_{0}, x_{n}\right)+q\left(x_{n-1}, x_{n}\right)} \frac{d r}{1+h(r)} \\
\leq \sum_{n=1}^{\infty} \int_{q\left(x_{0}, x_{n}\right)}^{q\left(x_{0}, x_{n}\right)+q\left(x_{n}, x_{n+1}\right)} \frac{d r}{1+h(r)} \\
<+\infty .
\end{aligned}
$$

This shows that

$$
\begin{aligned}
\int_{q\left(x_{0}, x_{1}\right)}^{\sum_{n=1}^{\infty} q\left(x_{n}, x_{n+1}\right)} & \frac{d r}{1+h(r)} \\
& \leq \sum_{n=1}^{\infty} \int_{q\left(x_{0}, x_{n}\right)}^{q\left(x_{0}, x_{n}\right)+q\left(x_{n}, x_{n+1}\right)} \frac{d r}{1+h(r)}<+\infty .
\end{aligned}
$$

By (95) we obtain that $\sum_{n=1}^{\infty} q\left(x_{n}, x_{n+1}\right)<+\infty$. Since $(X, \mathcal{U})$ is sequentially lower complete w.r.t. $(q, f \downarrow)$, by Lemma 26 , we get that there exists $\bar{x} \in X$ such that $q\left(x_{n}, \bar{x}\right) \rightarrow 0(n \rightarrow \infty)$, $\left\{x_{n}\right\}$ converges to $\bar{x}$, and, for any $y \in X$,

$$
\liminf _{n \rightarrow \infty} q\left(x_{n}, y\right) \leq p(\bar{x}, y) .
$$

Then there exists a subsequence $\left\{x_{n_{k}}\right\}$ such that

$$
\lim _{k \rightarrow \infty} q\left(x_{n_{k}}, y\right)=\liminf _{n \rightarrow \infty} q\left(x_{n}, y\right) \leq q(\bar{x}, y)
$$

Also, there exists a subsequence $\left\{x_{n_{k_{i}}}\right\}$ of $\left\{x_{n_{k}}\right\}$ such that

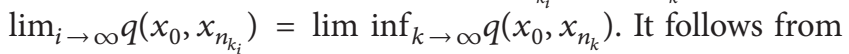
$q\left(x_{n}, \bar{x}\right) \rightarrow 0(n \rightarrow \infty)$ and $q\left(x_{0}, \bar{x}\right) \leq q\left(x_{0}, x_{n_{k_{i}}}\right)+q\left(x_{n_{k_{i}}}, \bar{x}\right)$ that $q\left(x_{0}, \bar{x}\right) \leq \lim _{i \rightarrow \infty} q\left(x_{0}, x_{n_{k_{i}}}\right)$. Then, by noting that $h$ is nondecreasing, we have

$$
\begin{aligned}
\liminf _{n \rightarrow \infty} p\left(x_{n}, y\right) & \leq \liminf _{k \rightarrow \infty} p\left(x_{n_{k}}, y\right) \\
& \leq \liminf _{i \rightarrow \infty} p\left(x_{n_{k_{i}}}, y\right) \\
& =\liminf _{i \rightarrow \infty} \int_{q\left(x_{0}, x_{n_{k_{i}}}\right)}^{q\left(x_{0}, x_{n_{k_{i}}}\right)+q\left(x_{n_{k_{i}}}, y\right)} \frac{d r}{1+h(r)} \\
& =\int_{\lim _{i \rightarrow \infty} q\left(x_{0}, x_{n_{k_{i}}}\right)}^{\lim _{i \rightarrow \infty} q\left(x_{0}, x_{n_{k_{i}}}\right)+\lim _{i \rightarrow \infty} q\left(x_{n_{k_{i}}}, y\right)} \frac{d r}{1+h(r)} \\
& \leq \int_{q\left(x_{0}, \bar{x}\right)}^{q\left(x_{0}, \bar{x}\right)+\lim _{i \rightarrow \infty} q\left(x_{n_{k_{i}}}, y\right)} \frac{d r}{1+h(r)} \\
& \leq \int_{q\left(x_{0}, \bar{x}\right)}^{q\left(x_{0}, \bar{x}\right)+q(\bar{x}, y)} \frac{d r}{1+h(r)}=p(\bar{x}, y) .
\end{aligned}
$$

Thus, $(X, \mathcal{U})$ is sequentially lower complete with respect to $p$ and $f$. The proof is completed.

Remark 35. By using Lemma 34 and Theorem 27, we can get coincidence point theorems and its equivalences for $(X, \mathscr{U})$ and $p$, which improve the results in $[29,30]$.

Remark 36. Let $(X, \mathfrak{I})$ be a sequentially complete $F$-type separated topological space (or equivalently, a uniform space) whose topology is generated by a family $\left\{q_{\lambda}\right\}_{\lambda \in \Lambda}$ of pseudometrics (see [15]), $\alpha: \Lambda \rightarrow(0,+\infty)$ a nondecreasing function, and $h:[0,+\infty) \rightarrow[0,+\infty)$ a nondecreasing function satisfying (95). Let $p_{\lambda}: X \times X \rightarrow[0,+\infty)$ be defined by

$$
p_{\lambda}(x, y)=\int_{q_{\lambda}\left(x_{0}, x\right)}^{q_{\lambda}\left(x_{0}, x\right)+q_{\lambda}(x, y)} \frac{d r}{1+h(r)}, \quad \lambda \in \Lambda,
$$

and let an extended real-valued function $p: X \times X \rightarrow$ $[0,+\infty]$ be defined by

$$
p(x, y)=\sup _{\lambda \in \Lambda} \alpha(\lambda) p_{\lambda}(x, y), \quad \forall(x, y) \in X \times X .
$$

Similar to the proof of Lemma 34, one can prove that $(X, \mathfrak{\Im})$ is sequentially lower complete w.r.t. $p$. Then, by using Theorem 27, we can get coincidence point theorems and its equivalences for $(X, \Im)$ and $p$, which improve the results in [32].

\section{Acknowledgments}

First, the authors are very grateful to the referees for their careful reading of the manuscript and a lot of valuable comments and suggestions, which greatly improve this paper. Next, this paper was supported by the National Natural Science Foundation of China (11171286, 11126344). 


\section{References}

[1] J. Caristi, "Fixed point theorems for mappings satisfying inwardness conditions," Transactions of the American Mathematical Society, vol. 215, pp. 241-251, 1976.

[2] J. Caristi, "Fixed point theory and inwardness conditions," in Applied Nonlinear Analysis, V. Lakshmikantham, Ed., pp. 479483, Academic Press, New York, NY, USA, 1979.

[3] I. Ekeland, "On the variational principle," Journal of Mathematics Analysis and Applications, vol. 47, pp. 324-353, 1974.

[4] I. Ekeland, "Nonconvex minimization problems," Bulletin of the American Mathematical Society, vol. 1, no. 3, pp. 443-474, 1979.

[5] A. Göpfert, C. Tammer, and C. Zălinescu, "On the vectorial Ekeland's variational principle and minimal points in product spaces," Nonlinear Analysis. Theory, Methods \& Applications, vol. 39, pp. 909-922, 2000.

[6] S. Al-Homidan, Q. H. Ansari, and J.-C. Yao, "Some generalizations of Ekeland-type variational principle with applications to equilibrium problems and fixed point theory," Nonlinear Analysis. Theory, Methods \& Applications, vol. 69, no. 1, pp. 126139, 2008.

[7] C. Bosch, A. García, and C. L. García, “An extension of Ekeland’s variational principle to locally complete spaces," Journal of Mathematical Analysis and Applications, vol. 328, no. 1, pp. 106108, 2007.

[8] S. S. Chang, Y. J. Cho, B. S. Lee, J. S. Jung, and S. M. Kang, "Coincidence point theorems and minimization theorems in fuzzy metric spaces," Fuzzy Sets and Systems, vol. 88, no. 1, pp. 119-127, 1997.

[9] J. S. Jung, S. S. Chang, Y. J. Cho, B. S. Lee, and S. M. Kang, "Common fixed point theorems and variational principle in generating spaces of quasi-metric family," Fuzzy Sets and Systems, vol. 102, no. 2, pp. 315-325, 1999.

[10] J. S. Jung, Y. J. Cho, S. M. Kang, and S.-S. Chang, "Coincidence theorems for set-valued mappings and Ekeland's variational principle in fuzzy metric spaces," Fuzzy Sets and Systems, vol. 79, no. 2, pp. 239-250, 1996.

[11] J. S. Jung, Y. J. Cho, S. M. Kang, B. S. Lee, and Y. K. Choi, "Coincidence point theorems in generating spaces of quasimetric family," Fuzzy Sets and Systems, vol. 116, no. 3, pp. 471479, 2000.

[12] S. Cobzaş, "Completeness in quasi-metric spaces and Ekeland variational principle," Topology and Its Applications, vol. 158, no. 8, pp. 1073-1084, 2011.

[13] J. X. Fang, "The variational principle and fixed point theorems in certain topological spaces," Journal of Mathematical Analysis and Applications, vol. 202, no. 2, pp. 398-412, 1996.

[14] A. G. Göpfert, H. Riahi, C. Tammer, and C. Zalinescu, Variational Methods in Partially Ordered Spaces, Springer, New York, NY, USA, 2003.

[15] A. H. Hamel, "Equivalents to Ekeland's variational principle in uniform spaces," Nonlinear Analysis. Theory, Methods \& Applications, vol. 62, no. 5, pp. 913-924, 2005.

[16] A. Hamel and A. Löhne, "A minimal point theorem in uniform spaces," in Nonlinear Analysis and Applications: To V. Lakshmikantham on His 80th Birthday, R. P. Argaval and D. O'Regan, Eds., vol. 1, pp. 577-593, Kluwer Academic Publisher, 2003.

[17] F. He and J. H. Qiu, "Generalization of Phelps' Lemma to bornological vector spaces," Acta Mathematica Scientia, vol. 31, no. 2, pp. 369-377, 2011 (Chinese).
[18] O. Kada, T. Suzuki, and W. Takahashi, "Nonconvex minimization theorems and fixed point theorems in complete metric spaces," Mathematica Japonica, vol. 44, no. 2, pp. 381-382, 1996.

[19] M. A. Khamsi, "Remarks on Caristi's fixed point theorem," Nonlinear Analysis. Theory, Methods \& Applications, vol. 71, no. 1-2, pp. 227-231, 2009.

[20] Z. Li, "Remarks on Caristi's fixed point theorem and Kirk's problem," Nonlinear Analysis. Theory, Methods \& Applications, vol. 73, pp. 3751-3755, 2010.

[21] L.-J. Lin and W.-S. Du, "Ekeland's variational principle, minimax theorems and existence of nonconvex equilibria in complete metric spaces," Journal of Mathematical Analysis and Applications, vol. 323, no. 1, pp. 360-370, 2006.

[22] L.-J. Lin and W.-S. Du, "Some equivalent formulations of the generalized Ekeland's variational principle and their applications," Nonlinear Analysis. Theory, Methods \& Applications, vol. 67, no. 1, pp. 187-199, 2007.

[23] L.-J. Lin and W.-S. Du, "On maximal element theorems, variants of Ekeland's variational principle and their applications," Nonlinear Analysis. Theory, Methods \& Applications, vol. 68, no. 5, pp. 1246-1262, 2008.

[24] J.-H. Qiu, "Ekeland's variational principle in locally convex spaces and the density of extremal points," Journal of Mathematical Analysis and Applications, vol. 360, no. 1, pp. 317-327, 2009.

[25] J. H. Qiu and F. He, "P-distances, q-distances and a generalized Ekeland's variational principle in uniform spaces," Acta Mathematica Sinica (English Series), vol. 28, no. 2, pp. 235-254, 2012.

[26] T. Suzuki, "Generalized distance and existence theorems in complete metric spaces," Journal of Mathematical Analysis and Applications, vol. 253, no. 2, pp. 440-458, 2001.

[27] D. Tataru, "Viscosity solutions of Hamilton-Jacobi equations with unbounded nonlinear terms," Journal of Mathematical Analysis and Applications, vol. 163, no. 2, pp. 345-392, 1992.

[28] C.-W. Wong, "A drop theorem without vector topology," Journal of Mathematical Analysis and Applications, vol. 329, no. 1, pp. 452-471, 2007.

[29] C.-K. Zhong, "A generalization of Ekeland's variational principle and application to the study of the relation between the weak P.S. condition and coercivity," Nonlinear Analysis. Theory, Methods \& Applications, vol. 29, no. 12, pp. 1421-1431, 1997.

[30] J. Zhu, C. K. Zhong, and Y. J. Cho, "Generalized variational principle and vector optimization," Journal of Optimization Theory and Applications, vol. 106, no. 1, pp. 201-217, 2000.

[31] J. Zhu and S. J. Li, "Generalization of ordering principles and applications," Journal of Optimization Theory and Applications, vol. 132, no. 3, pp. 493-507, 2007.

[32] J. Zhu, C.-K. Zhong, and G.-P. Wang, "An extension of Ekeland's variational principle in fuzzy metric space and its applications," Fuzzy Sets and Systems, vol. 108, no. 3, pp. 353-363, 1999.

[33] T. L. Hicks and B. E. Rhoades, "Fixed point theory in symmetric spaces with applications to probabilistic spaces," Nonlinear Analysis. Theory, Methods \& Applications, vol. 36, no. 3, pp. 331344, 1999.

[34] T. L. Hicks, "Fixed point theorems for $d$-complete topological spaces. I," International Journal of Mathematics and Mathematical Sciences, vol. 15, no. 3, pp. 435-439, 1992.

[35] J. Zhu, Y. J. Cho, and S. M. Kang, "Equivalent contractive conditions in symmetric spaces," Computers \& Mathematics with Applications, vol. 50, no. 10-12, pp. 1621-1628, 2005. 
[36] R. H. Haghi, Sh. Rezapour, and N. Shahzad, "Some fixed point generalizations are not real generalizations," Nonlinear Analysis. Theory, Methods \& Applications, vol. 74, no. 5, pp. 1799-1803, 2011.

[37] H. Hogbe-Nlend, Bornologies and Functional Analysis, NorthHolland, Amsterdam, The Netherlands, 1977.

[38] I. L. Reilly, P. V. Subrahmanyam, and M. K. Vamanamurthy, "Cauchy sequences in quasipseudometric spaces," Monatshefte für Mathematik, vol. 93, no. 2, pp. 127-140, 1982.

[39] O. Kaleva and S. Seikkala, “On fuzzy metric spaces," Fuzzy Sets and Systems, vol. 12, no. 3, pp. 215-229, 1984. 


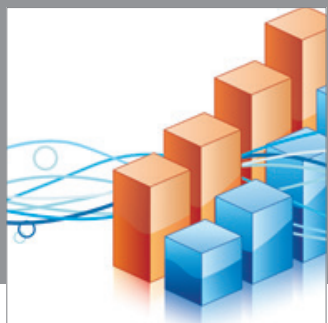

Advances in

Operations Research

mansans

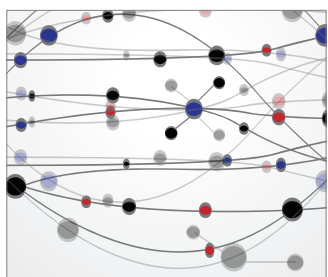

The Scientific World Journal
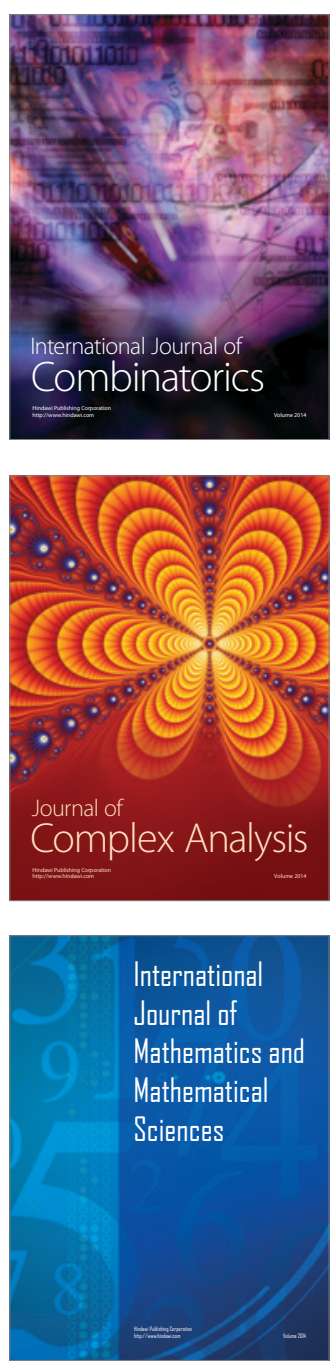
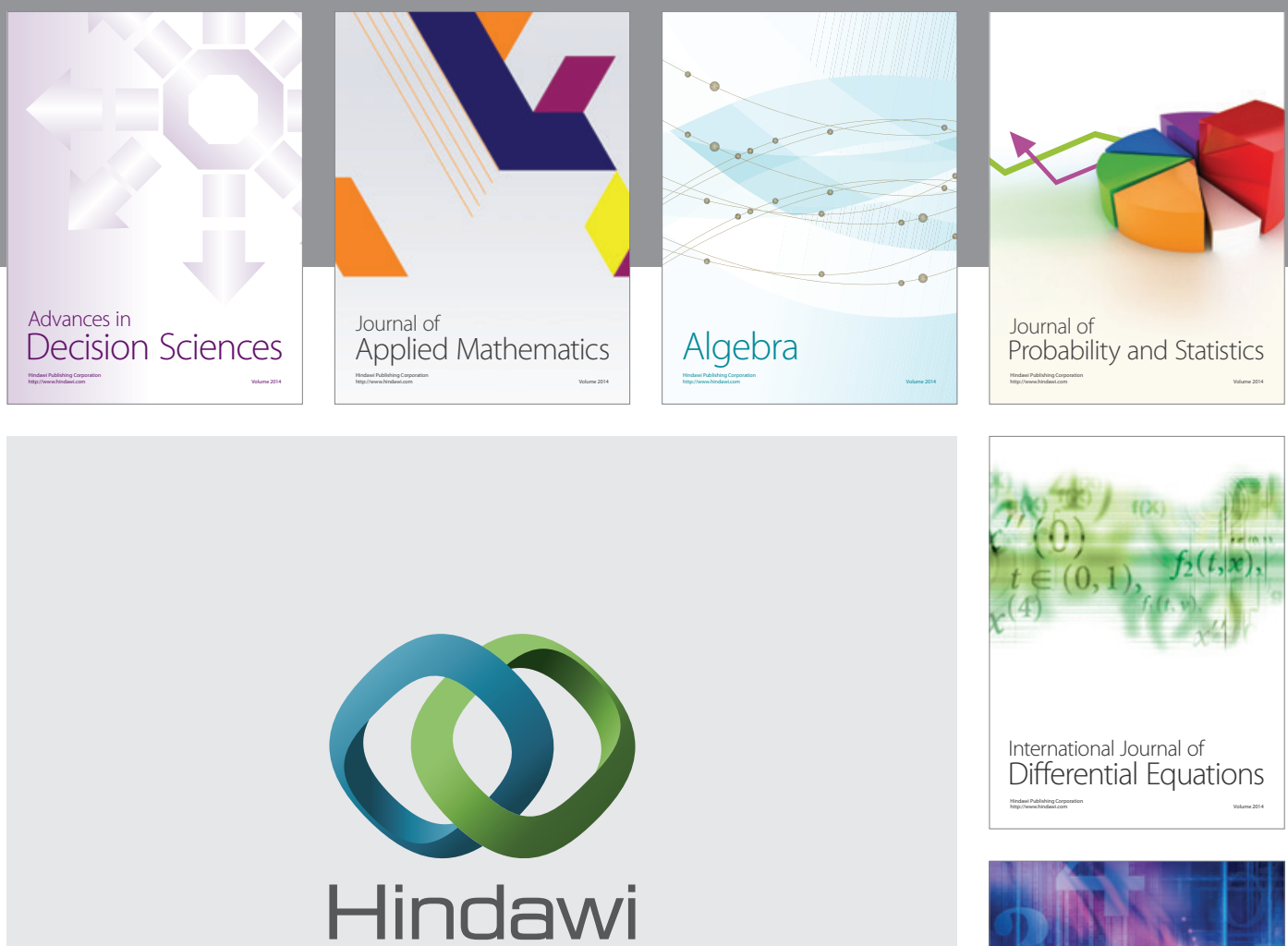

Submit your manuscripts at http://www.hindawi.com
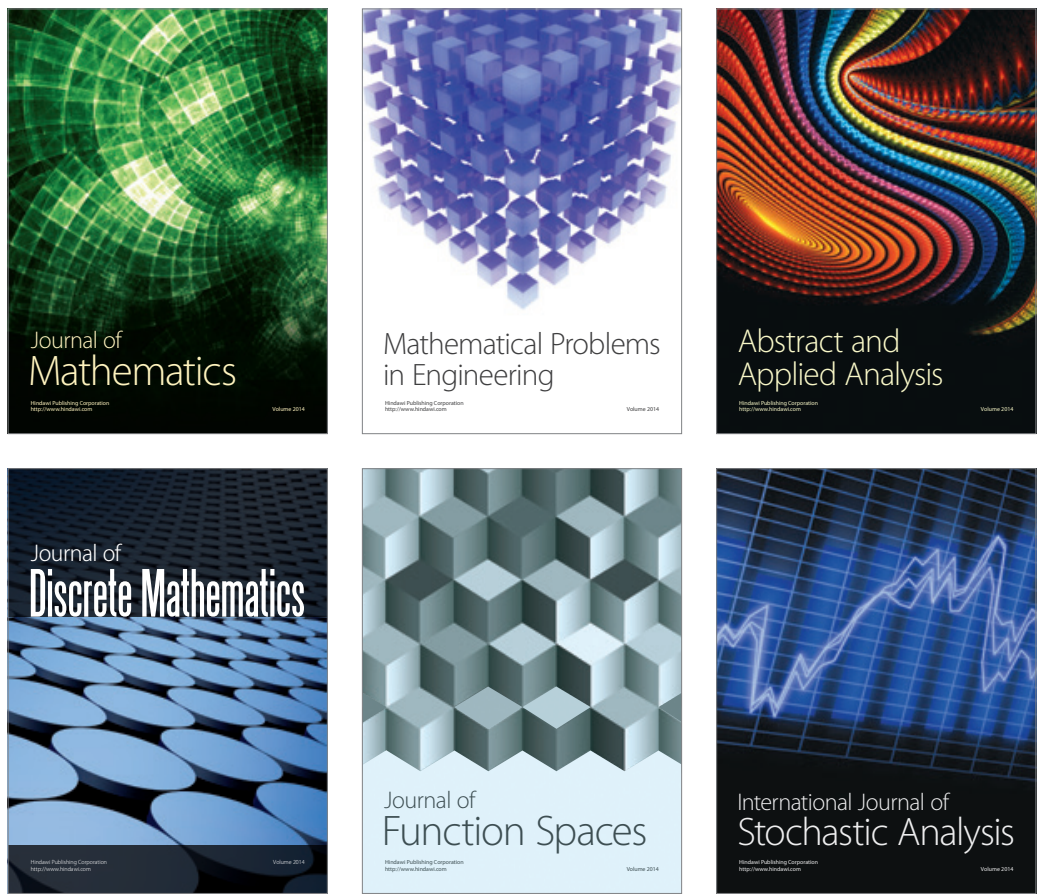

Journal of

Function Spaces

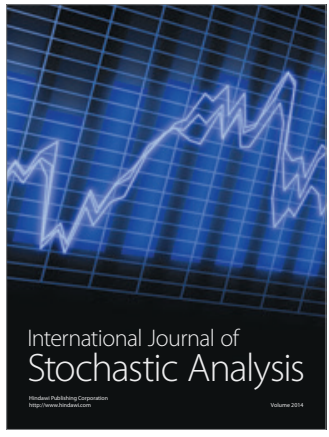

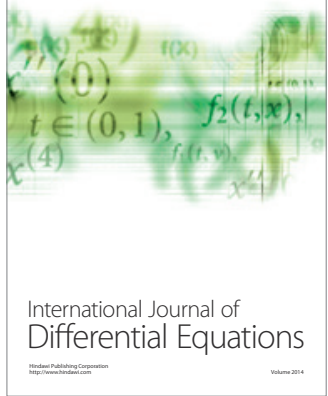
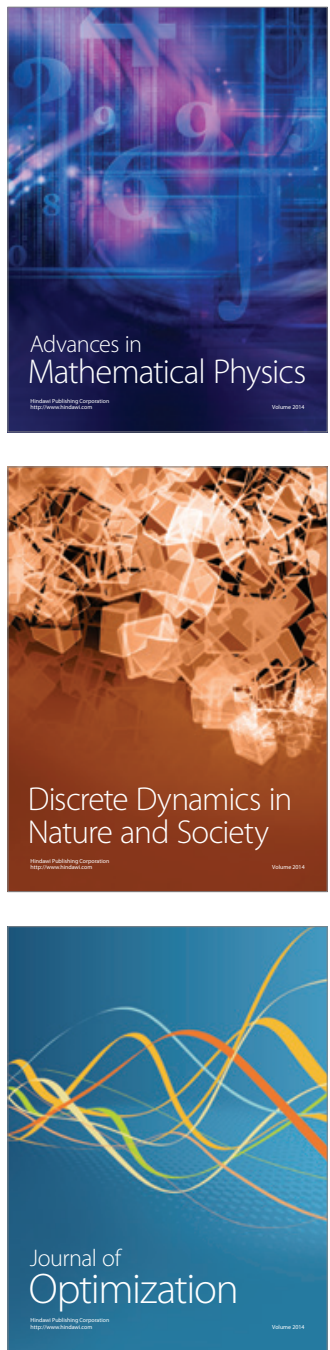